OPEN ACCESS

Edited by:

Luis A. J. Mur,

Aberystwyth University,

United Kingdom

Reviewed by:

Upinder S. Gill,

University of Florida, United States

Andrea Sanchez Vallet,

ETH Zürich, Switzerland

*Correspondence:

Robert Brueggeman

robert.brueggeman@ndus.edu

Specialty section:

This article was submitted to

Plant Breeding,

a section of the journal

Frontiers in Plant Science

Received: 25 July 2018 Accepted: 19 November 2018

Published: 05 December 2018

Citation:

Sharma Poudel R, Al-Hashel AF, Gross T, Gross $P$ and Brueggeman $R$

(2018) Pyramiding rpg4-

and Rpg1-Mediated Stem Rust

Resistance in Barley Requires

the Rrr1 Gene for Both to Function.

Front. Plant Sci. 9:1789.

doi: 10.3389/fpls.2018.01789

\section{Pyramiding rpg4- and Rpg1-Mediated Stem Rust Resistance in Barley Requires the Rrr1 Gene for Both to Function}

\author{
Roshan Sharma Poudel, Abdullah F. Al-Hashel, Thomas Gross, Patrick Gross and \\ Robert Brueggeman*
}

Department of Plant Pathology, North Dakota State University, Fargo, ND, United States

Stem rust, caused by Puccinia graminis f. sp. tritici (Pgt) is an economically important disease of wheat and barley. Rpg1 is the only resistance gene deployed in Midwestern US barley varieties and provides remarkable resistance to most North American races, except Pgt race QCCJB. Rpg1 is also ineffective against Pgt race TTKSK and its lineage that originated in Africa. The barley rpg4-mediated resistance locus (RMRL) conferring resistance to Pgt races QCCJB and TTKSK was isolated from line Q21861, which is resistant to all known Pgt races due to Rpg1 and RMRL. To develop elite barley varieties $R M R L$ was pyramided into the varieties, Pinnacle and Conlon (both contain Rpg1), producing the near isogenic lines (NILs), Pinnacle RMRL-NIL (PRN) and Conlon RMRL-NIL (CRN). The CRN was resistant to Pgt races QCCJB (RMRL specific) and HKHJC (Rpg1 specific) at the seedling stage and Pgt race TTKSK (RMRL specific) at the adult stage. In contrast, PRN was susceptible to QCCJB and HKHJC at the seedling stage and TTKSK at the adult stage. Interestingly, PRN's susceptibility to QCCJB and HKHJC showed that RMRL was non-functional in the Pinnacle background but its presence also suppressed Rpg1-mediated resistance. Thus, in the absence of a gene/s found in the Q21861 background, Rpg1 becomes non-functional if RMRL is present, suggesting that another polymorphic gene, that we designated Rrr1 (required for rpg4mediated resistance 1), is required for RMRL resistance and Rpg1-mediated resistance in the presence of RMRL. Utilizing a PRN/Q21861 derived recombinant inbred line (RIL) population, Rrr1 was delimited to a $\sim 0.5 \mathrm{MB}$ physical region, slightly proximal ( 1.8 MB) of RMRL on barley chromosome $5 \mathrm{H}$. A second gene, designated required for $\underline{R} p \mathrm{1}$ mediated resistance 2 (Rrr2), with duplicate gene action to Rrr1 in Rpg1-mediated resistance function, was genetically delimited to a physical region of $\sim 0.7 \mathrm{MB}$, slightly distal ( 3.1 MB) to Rpg1 on the short arm of barley chromosome $7 \mathrm{H}$. Thus, Rrr1 is required for RMRL resistance and Rrr1 or Rrr2 is required for functional Rpg1-mediated resistance in the presence of the RMRL introgression. Candidate Rrr1 and Rrr2 genes were identified that need to be considered when pyramiding Rpg1 and RMRL in barley.

Keywords: barley, stem rust resistance, genetic mapping, PCR-GBS, iSelect marker 


\section{INTRODUCTION}

The obligate biotrophic fungus Puccinia graminis f. sp. tritici Eriks. and E. Henn. (Pgt) is the causal agent of stem rust (or black rust) in bread wheat (Triticum aestivum L.) and barley (Hordeum vulgare L.). Historically, stem rust epidemics caused devastating yield losses of wheat and barley in several parts of the world, including the northern Great Plains of the United States (Roelfs, 1992; Steffenson, 1992). The northern Great Plains is a stem rust prone area that regularly suffered stem rust epidemics in barley prior to the 1940s (Steffenson, 1992). In 1942, the barley variety Kindred was released as the first commercial barley containing the stem rust resistance gene Rpg1 (Resistance to P. graminis 1) (Steffenson, 1992) and since then, barley-breeding programs in the upper Midwest of the United States have fixed the Rpg1 gene in their lines, which originally came from either cv. Peatland (CIho 5267), Chevron (Ciho 111) or Kindred (Ciho 6969) (Steffenson, 1992; Jin et al., 1994a; Zhou et al., 2014). Thus, for nearly 80 years, Rpg1 has been the only source of durable resistance in barley and has maintained its effectiveness against the majority of isolates making up the North American Pgt populations.

In 1989, a new race of Pgt, designated as QCC [later designated as QCCJB (Jin et al., 2008)] was identified in North Dakota (ND) that was virulent on barley cultivars carrying Rpg1 (Roelfs et al., 1991). The Pgt race QCCJB became the most prevalent race in the northern US, threatening regional commercial barley varieties with potential stem rust epidemics that had not been experienced for nearly 50 years (Roelfs et al., 1993). To tackle this threat, Jin et al. (1994a) screened 18,000 barley accessions from the USDA National Small Grains collection (Aberdeen, ID) and identified the unimproved barley line Q21861 as a remarkable source of resistance against QCCJB. Genetic studies utilizing several biparental populations derived from Q21861 and different susceptible barley lines characterized a temperature sensitive and recessive gene designated rpg4 in the line Q21861 conferring resistance to Pgt race QCCJB (Jin et al., 1994b). The rpg4 gene was mapped to the sub-telomeric region of the long arm of barley chromosome 5H (Borovkova et al., 1995). Q21861 also contains a dominant resistance gene that confers resistance to the rye stem rust pathogen $P$. graminis f. sp. secalis $(P g s)$ isolate 92-MN-90 that was originally reported to co-segregate with rpg4, and was designated as Rpg5 (Sun et al., 1996; Brueggeman et al., 2008). A map-based cloning approach identified the dominant rye stem rust resistance gene Rpg5 as a nucleotidebinding site leucine-rich repeat-serine/threonine protein kinase (NBS-LRR-STPK) domain protein (Brueggeman et al., 2008). The original high-resolution map determined that Rpg5 was independent of, but tightly linked to rpg4 and colocalized to a $70 \mathrm{~kb}$ physical region (Brueggeman et al., 2008). Utilizing the high-resolution mapping populations and additional single nucleotide polymorphism (SNP) markers, the rpg4/Rpg5 locus was refined. In two of the high-resolution populations the genes colocalized to the $70 \mathrm{~kb}$ region containing the $R p g 5$ rye stem rust resistance gene and was delimited to three tightly linked genes within the region; the NBS-LRR gene HvRgal, the NBSLRR-STPK gene Rpg5, and an actin depolymerizing factor-like
(Adf) gene, HvAdf3 (Wang et al., 2013). Post-transcriptional gene silencing utilizing barley stripe mosaic virus (BSMV) virusinduced gene silencing (VIGS) of each of the genes delimited to the rpg4/Rpg5 locus determined that all three genes, HvRgal, $R p g 5$ and $H v A d f 3$ are required together to confer rpg4-mediated stem rust resistance. Thus, this complex of genes required for wheat stem rust resistance was designated the rpg4-mediated resistance locus (RMRL). However, a study of multiple alleles of Rpg5, HvRgal and HvAdf3 determined that Rpg5 is the only gene that contains polymorphisms that explain resistancevs-susceptibility, therefore, Rpg5 appears to be the resistance gene with functional polymorphism at the locus, yet HvRgal and $H v A d f 3$ are required for resistance (Arora et al., 2013). Interestingly, the major allele found in cultivated and wild barley lines collected from around the world (Arora et al., 2013; Steffenson et al., 2017) contain a PP2C domain in place of the Rpg5-STPK domain in most Pgt race QCCJB susceptible barley lines that result in the loss of RMRL-mediated resistance. Thus, markers based on this polymorphism are used to track RMRL introgression via marker-assisted selection (Derevnina et al., 2014).

Currently, the highly virulent $P g t$ race TTKSK (also known as Ug99) and its lineage pose an alarming threat to global wheat and barley production and world food security (Steffenson and Jin, 2006; Singh et al., 2011; Steffenson et al., 2017). Race TTKSK was first reported in 1999 from wheat fields in Uganda, Africa (Pretorius et al., 2000). The extensive monitoring of this race showed a rapid spread of TTKSK and its evolving lineage throughout other countries in Africa and jumped the red sea into Asia (Singh et al., 2011). The varieties grown on approximately $80-95 \%$ of wheat acreage world-wide and the majority of breeding materials were found to be moderately to highly susceptible to TTKSK and its lineage (Singh et al., 2006, 2011). Recently, Steffenson et al. (2017) reported 96\% of 2,913 barley accessions, including those containing Rpg1, were extremely vulnerable to this evolving Pgt race. The reliance on a single resistance gene in the Upper Midwestern US and identification of North American race QCCJB, and the threat of the emergence of African race TTKSK and its lineage leaves barley production in the major barley production region of US and the world vulnerable to potential stem rust epidemics. In barley, RMRL is the only well characterized locus that provides resistance to QCCJB, TTKSK and its lineage (Steffenson et al., 2009), thus, introgression of RMRL into commercially grown barley varieties is important to minimize the risk of stem rust races that have overcome the remarkably durable Rpg1-mediated resistance.

The primary goal of barley-breeding programs is to release varieties that are high yielding, have good quality, are stable across dynamic environments and contain resistance to important pathogens or pests (Horsley et al., 2009). Conlon and Pinnacle are recommended malting barley varieties grown in the Upper Midwestern United States (United States Department of Agriculture [USDA], 2012). Like all Midwestern barley varieties, Conlon and Pinnacle are known to contain only the Rpg1mediated stem rust resistance gene, thus, are prone to potential stem rust epidemics from $P g t$ races like QCCJB or TTKSK if 
or when it was to arrive in the US. Thus, a marker assisted backcrossing scheme was initiated to introgress RMRL into these lines. The Conlon RMRL-near isogenic line (NIL) and Pinnacle RMRL-NIL (PRN) carrying both Rpg1 and the RMRL were developed via marker-assisted selection. Field trials in Kenya using Pgt race TTKSK showed that only the Conlon RMRL-NIL (CRN) gained resistant to $P g t$ race TTKSK at the adult plant stage in the field but the PRN did not. Corresponding with the adult plant reactions in the field, the CRN exhibited resistance to Pgt race QCCJB while the PRN was susceptible to Pgt race QCCJB in growth chamber seedling resistance assays. Intriguingly, a later growth chamber assay of the PRN using the Rpg1 avirulent Pgt race HKHJC showed that the PRN not only lacked RMRLmediated resistance but had also lost Rpg1-mediated stem rust resistance, even though the Rpg1 gene was fixed.

Arora et al. (2013) sequenced Rpg5 alleles from a diverse set of barley accession and categorized them into four resistant and four susceptible groups based on polymorphisms present in the functional Rpg5 and non-functional rpg5 alleles. Pinnacle and Conlon, carry the non-functional rpg5 allele that contains a C-terminal protein phosphatase 2C (PP2C) integrated domain in place of the C-terminal protein kinase domain present in the functional Rog 5 alleles. Thus, these alleles belong to either the group 2 or group 3 susceptible genotypes as described by Arora et al. (2013). A combination of Rpg5-LRR/STPK and Rpg5LRR/PP2C specific sequence tag site polymerase chain reaction (PCR) markers were used for marker assisted selection to confirm the presence of full-length Q21861 like Rpg5-STPK alleles in elite barley backgrounds from breeding programs around the world, including our introgression into the Conlon and Pinnacle backgrounds. The single Rpg5 marker was considered sufficient to track the entire RMRL containing HvRga1, Rpg5 and HvAdf3 as genotyping of over 5,000 recombinant gametes did not identify a single recombinant separating the three genes (Brueggeman et al., 2008; Wang et al., 2013) and the HvRgal and HvAdf3 genes are highly conserved across resistant and susceptible genotypes (Arora et al., 2013). Also, the Rpg1 gene in all resistance sources in Midwestern breeding programs including the NDSU breeding program that produced Conlon and Pinnacle are identical to the Rpg1 gene found in Q21861, thus, Rpg1 is fixed in all lines utilized in the pyramiding scheme. However, despite the presence of the Q21861 like RMRL, and intact Rpg1 in the PRN, the loss of resistance to both Rpg1 and RMRL avirulent Pgt races HKHJC and QCCJB, respectively, at the seedling stage and TTKSK at the adult stage in the field should be due to a gene/s that are required for resistance but are non-functional in the line Pinnacle. These data suggest the existence of other gene/s required for Rpg1 and/or RMRL stem rust resistance with natural polymorphism in the primary barley germplasm pool which must be considered when pyramiding these two effective stem rust resistance genes that when present together confer resistance to all known stem rust races.

The aim of this study was to identify the region of the barley genome that segregates for genes required for effective stem rust resistance when RMRL and Rpg1 are combined in different barley backgrounds. To this end a $\mathrm{F}_{4: 5}$ recombinant inbred line (RIL) mapping population was developed from the cross between the PRN/Q21861 using single-seed descent to map the other genes required for resistance since both RMRL and Rpg1 are fixed in the population thus the only genes to segregate would be the other genes required for resistance. Utilizing this biparental mapping population, two genes, Rrr1 (required for $r p g 4$-mediated resistance 1) and Rrr2 (required for Rpg1mediated resistance 2) were mapped to barley chromosomes $5 \mathrm{H}$ and $7 \overline{\mathrm{H}}$, respectively. The genetic and phenotypic analysis revealed that a functional $R r r 1$ is required for RMRL resistance yet neither gene is required for Rpg1-mediated resistance in the absence of the RMRL. However, when RMRL is added to the genetic background then Rrr1 or Rrr 2 are required for the Rpg1mediated resistance to function. Thus, based on results from this study, we hypothesize that the introgression of RMRL requires Rrr1 and to pyramid RMRL with Rpg1 a functional Rrr1 or Rrr2 is necessary to maintain the $R p g 1$-mediated resistance function.

\section{MATERIALS AND METHODS}

\section{Development of Pinnacle and Conlon RMRL-NIL}

The Pinnacle and CRNs were originally developed to provide elite regional (Upper Midwestern US) adapted germplasm that contained broad resistance to all known stem rust races. The Pinnacle and CRNs were developed via a backcrossing and marker assisted selection scheme. Pinnacle, a two-row malting cultivar developed by the NDSU barley-breeding program and released by the North Dakota Agricultural Experiment Station in 2006, was crossed with the unimproved two-row barley line Q21861 as the RMRL donor. Conlon is also a two-row malting barley variety developed by the NDSU barley-breeding program that was an earlier release by the North Dakota Agricultural Experiment Station in 1996. Conlon was crossed with the Harrington $x$ Q21861 $\mathrm{BC}_{3} \mathrm{~F}_{2}$ line designated HQ1 as the RMRL donor. The HQ1 line was developed by backcrossing to the recurrent parent Harrington to the $\mathrm{BC}_{3}$ generation using rpg5PP2C allele specific STS markers (primers R5iN-F2 and PP2CR3) for MAS to insure introgression of RMRL into a Northwest US and Canadian adapted malting barley variety (Brueggeman et al., 2008). The susceptible variety Harrington used to develop the HQ1 line is a two-row malting variety that contains no known stem rust resistance genes and was developed at the University of Saskatchewan (Harvey and Rossnagel, 1984).

The Pinnacle and Conlon RMRL introgression was accomplished by backcrossing $\mathrm{BC}_{1} \mathrm{~F}_{1}$ derived individuals at each generation to the recurrent elite malting background that were subsequently selfed to generate $F_{2}$ progeny. Approximately 12-15 $\mathrm{F}_{2}$ individuals from each cross were genotyped using the RMRL specific markers (described below in Marker assisted selection for $R M R L$ ) and progeny homozygous for RMRL were selected. The homozygous rpg5-STPK/RMRL selected $\mathrm{F}_{2}$ individuals from the Pinnacle and Conlon backcrosses were again crossed to the recurrent parents. The backcrossing, selfing and marker assisted selection for RMRL was repeated twice for Pinnacle and thrice for Conlon to develop, $\mathrm{BC}_{3} \mathrm{~F}_{1}$ Pinnacle and $\mathrm{BC}_{4} \mathrm{~F}_{1}$ Conlon, individuals, respectively. The final 
backcrossed individual was selfed to identify $\mathrm{BC}_{3} \mathrm{~F}_{3}$ Pinnacle (Q21861/4* Pinnacle $\mathrm{F}_{3}$ ) and $\mathrm{BC}_{4} \mathrm{~F}_{3}$ Conlon (Q21861/4*Conlon $\mathrm{F}_{3}$ ) NILs containing both Rpg1 and RMRL in the homozygous state.

\section{Marker Assisted Selection for RMRL}

Two previously designed dominant markers, Rpg5-LRK and Rpg5-LRK/PP2C were used to detect the presence of functional Rpg5-STPK allele and absence of the rpg5-PP2C allele, respectively (Derevnina et al., 2014). Genomic DNA (gDNA) was extracted from leaf samples using the protocol described in Richards et al. (2016). PCRs were performed using a Mastercycler Pro programmable thermocycler (Eppendorf, Hauppauge, NY, United States) programmed for $95^{\circ} \mathrm{C}$ for $4 \mathrm{~min}$; followed by 35 cycles of $95^{\circ} \mathrm{C}$ for $30 \mathrm{~s}, 62^{\circ} \mathrm{C}$ for $1 \mathrm{~min}$, and $72^{\circ} \mathrm{C}$ for $1 \mathrm{~min}$; followed by $72^{\circ} \mathrm{C}$ for $5 \mathrm{~min}$. PCR amplifications were performed in a $20 \mu \mathrm{l}$ reaction volume containing $10 \mathrm{ng}$ of template gDNA, $1 \times$ GoTaq $^{\circledR}$ Flexi Buffer (Promega, United States), $1 \mathrm{mM} \mathrm{MgCl}_{2}$, $0.15 \mathrm{mM}$ dNTP mix, $0.4 \mu \mathrm{M}$ of each forward and reverse primer, $1.25 \mu \mathrm{GoTaq}^{\circledR}$ DNA polymerase. $\mathrm{BC}_{n} \mathrm{~F}_{2}$ lines were selected for further backcrossing if they amplified a 1,046-bp fragment from Rpg5-STPK alleles utilizing Rpg5-LRK markers but had no amplification for the rpg5-PP2C allele as indicated by an $840 \mathrm{bp}$ amplicon when utilizing the Rpg5-LRK/PP2C marker.

\section{Development of RIL Mapping Population}

To develop the PRN/Q21861 RIL mapping population with fixed RMRL and Rpg1 genes to map the gene/s involved in RMRL- and Rpg1-mediated resistance pathway, the PRN (RMRL+/Rpg1+) was crossed with barley accession Q21861 (RMRL+/Rpg1+). The $F_{1}$ generated from the PRN/Q21861 cross was self-fertilized and $F_{2}$ individuals were advanced to the $F_{4: 5}$ generation by single-seed descent (Brim, 1966) to generate a 126 individual RIL population. At the $\mathrm{F}_{2}$ stage, 94 of the 126 individuals that were advanced to develop the RIL were phenotyped using Pgt race QCCJB to determine the mode of inheritance of $\mathrm{Rrr} 1$ (rust inoculation explained in rust assay section). These individuals were assayed at 14 days post inoculations and later transplanted from cones to 6 -inch pots to grow along with other remaining $\mathrm{F}_{2}$ individuals to self-fertilize for RIL population advancement.

\section{Stem Rust Disease Phenotypic Assays Field Assay}

To identify NILs exhibiting TTKSK resistance, four $\mathrm{F}_{2}$ derived PRN individuals and $12 \mathrm{~F}_{2}$ derived $\mathrm{CRN}$ individuals were evaluated in the International Stem Rust Nursery at the Kenyan Agricultural Research Institute in Njoro, Kenya during the 2015 field season (Supplementary Table S1). The parental lines (Q21861, Pinnacle and Conlon), susceptible control (Steptoe) and the spreader rows for rust inoculum were planted as described in Zurn et al. (2014). The entries were planted in two replications and the screening was done at the adult plant stage. The modified Cobb scale was used to record the disease severity of stem rust (Peterson et al., 1948; McIntosh et al., 1995). Based on the size and type of the uredinia, the infection response (IR) were categorized into resistant ( $\mathrm{R}$; small uredinia surrounded by chlorosis or necrosis), moderately resistant (MR; medium sized uredinia surrounded by chlorosis or necrosis), moderately susceptible (MS; medium to large size uredinia without chlorosis or necrosis), and susceptible (S; large compatible uredinia without chlorosis or necrosis), or intermediate of any two categories (MRMS, MSS, SMS) (Roelfs, 1992; McIntosh et al., 1995; Yu et al., 2011). The weighted score of modified Cobb scale were taken as coefficient of infection as explained by $\mathrm{Yu}$ et al. (2011). The conversion of modified Cobb scale is explained in Supplementary Table S1.

\section{Growth Chamber Assays \\ Experimental design}

To confirm the disease response observed in the field, one of the resistant CRN individual (FAR14-94A-1) and one of the susceptible PRN individual (FAR14-1-1) were selected for seedling assays in the growth chamber using Pgt race QCCJB and HKHJC. Pgt isolate QCCJB is virulent on Rpg1 but avirulent on RMRL; and HKHJC is virulent on RMRL but avirulent on Rpg1. Any NIL resistant to both isolates suggests the presence of a functional Rpg1 and RMRL ( $r p g 4 / R p g 5$ genes). The parental lines, Q21861, HQ1, Pinnacle, Conlon and the susceptible controls, Harrington and Steptoe were planted for each disease reaction assay. Each entry was planted in seven cones and randomly distributed in a $12 \times 7$ rack.

Seedling assay was also conducted on $\mathrm{F}_{2}$ progeny lines and the PRN/Q21861 $\mathrm{F}_{4: 5}$ RILs using Pgt race QCCJB and the further advanced $\mathrm{F}_{5: 6}$ RIL population was screened using the two Pgt races QCCJB (avirulent on RMRL and virulent on Rpg1) and HKHJC (virulent on RMRL and avirulent on Rpg1). For the assay using $\mathrm{F}_{2}$ progenies, $94 \mathrm{~F}_{2}$ progenies were randomly selected and grown in 6-inch plastic cones with one seed per cone along with the PRN and Q21861 parents. The phenotypic segregation for Pgt race QCCJB in the $\mathrm{F}_{2}$ population segregated in a 3:1 ratio (resistance to susceptibility) showing a qualitative/single gene segregation. To map the single gene qualitative trait in the RIL population, two independent experimental replications of the PRN/Q21861 F $\mathrm{F}_{4: 5}$ RILs were inoculated with Pgt race QCCJB or HKHJC in growth chamber assay. For each independent experiment, two replication of each RIL in separate cones containing two individual seedlings were planted and arranged in a randomized complete block design. For each rep of the 126 RIL population the parental lines Q21861 and PRN, as well as Pinnacle, CRN, Conlon, Morex, Steptoe and Harrington were assayed as controls. Morex is a six-row malting barley cultivar release by the University of Minnesota that carries Rpg1 (Kleinhofs et al., 1993). Harrington and Steptoe are wheat stem rust susceptible lines which do not harbor any known stem rust resistance genes (Brueggeman et al., 2008). For the assay using HKHJC, results were only available from a single replication of the first experiment and both replications of the second experiment.

\section{Stem rust inoculation}

After planting, the rack containing cones with seeds were transferred to a growth chamber (Model 7301-75-2; Caron, Marietta, OH, United States) set at a 16/8 h light/dark cycle and a day/night temperature of $21 / 18^{\circ} \mathrm{C}$. When the primary leaves were 
fully expanded and the secondary leaf was still at the whorl stage (7-8 days post sowing), the seedlings were inoculated with $P g t$ race QCCJB or HKHJC using the protocol previously described by Steffenson et al. (2009) and moved to a humidity chamber. After $18 \mathrm{~h}$ in a dark humidity chamber at $100 \%$ relative humidity, the inoculated plants were moved back to the growth chamber set at the previously described growing condition for 14 days. At 14 days post inoculation (DPI), the infection types (ITs) were assessed using a modified 0-4 rating scale established for barley (Stakman et al., 1962; Steffenson et al., 2009). The scores were converted to a quantitative score using a conversion scale devised by Zhou et al. (2014). The conversion scale is explained in Supplementary Table S2.

\section{Statistical Analysis}

To classify the individual as resistant or susceptible, the quantitative scores of ITs were obtained using the conversion scale of Zhou et al. (2014). Any individual with a score greater than 3 was classified as susceptible. The Pearson's chi-square $\left(\chi^{2}\right)$ goodness of fit test statistic was used to evaluate the independent segregation of resistance to susceptibility in the $F_{2}$ and $\mathrm{F}_{4: 5}$ RIL populations, for single or two gene segregation. The test result in the $F_{2}$ population was used to determine the nature of inheritance of the gene/s. Since only Pgt race QCCJB was used to assay the $F_{2}$ population, the inheritance of Rrrl was determined as a single gene required for RMRLmediated resistance, but the inheritance of Rrr2 was not determined. The $p$-value associated with Pearson's $\chi^{2}$ statistics was obtained using the CHISQ.TEST function in Microsoft Excel.

\section{gDNA Extraction for PCR-GBS}

Polymerase chain reaction-genotyping by sequencing (PCRGBS) was used to genotype the PRN/Q12861 $\mathrm{F}_{4: 5}$ RIL population and the parental lines PRN and Q21861, as well as Pinnacle. The gDNA used for PCR-GBS was isolated using a modified version of the 96-well plate extraction methods described by Ivanova et al. (2008) (protocol obtained from Dr. Xuehui Li's lab, Department of Plant Sciences, NDSU). An $\sim 3 \mathrm{~cm}$ piece leaf tissue from each individual were collected in 96-well $2.2 \mathrm{ml}$ deep well plates (VWR, PA, United States) and lyophilized for $24 \mathrm{~h}$ at $-40^{\circ} \mathrm{C}$. Two $4 \mathrm{~mm}$ stainless steel grinding balls (VWR, PA, United States) were added to each well and the samples were powdered using a Mixer Miller Type 301 tissue grinder (Retsch Gmbh \& Co. KG, Germany) set at a frequency of 20/s for 3-6 min. The powdered tissue samples were homogenized in $500 \mu \mathrm{l}$ extraction buffer $(100 \mathrm{mM}$ Tris base $\mathrm{pH} 8.0,50 \mathrm{mM}$ EDTA pH 8.0, $500 \mathrm{mM} \mathrm{NaCl}, 1.25 \% \mathrm{SDS}$ ) and incubated at $65^{\circ} \mathrm{C}$ for $30 \mathrm{~min}$ with brief vortexing every $10 \mathrm{~min}$. The plates were incubated at $-20^{\circ} \mathrm{C}$ for $10 \mathrm{~min}$, then $166 \mu \mathrm{l}$ of precipitation solution (5M ammonium acetate) was added to each sample. The samples were briefly vortexed and transferred back to $-20^{\circ} \mathrm{C}$ for $10 \mathrm{~min}$. The plates were centrifuged at room temperature at 4,000 RPM (Revolution Per Minute) for $25 \mathrm{~min}$. About $400 \mu \mathrm{l}$ of supernatant was transferred to a fresh 96-well $2.2 \mathrm{ml}$ deep well plate containing $600 \mu \mathrm{l}$ of DNA binding solution (6M Guanidine$\mathrm{HCl}, 63 \%$ alcohol) and mixed by pipetting $3-5$ times. The mixed samples were transferred to a AcroPrep ${ }^{\mathrm{TM}}$ DNA filter plate stack (part\# 8132, Pall Corporation, Port Washington, NY) set over 96-well $2.2 \mathrm{ml}$ deep well plates and centrifuged (3,000 RPM) for $10 \mathrm{~min}$ at room temperature. The eluents were discarded after centrifugation followed by two rounds of filter wash using $800 \mu \mathrm{l}$ of wash solution (10 mM Tris pH 8.0, $1 \mathrm{mM}$ EDTA $\mathrm{pH}$ 8.0, $50 \mathrm{mM} \mathrm{NaCl}, 67 \%$ ethanol). The remnant wash solution after the final wash was removed by centrifugation for $10 \mathrm{~min}$ at 3,000 RPM. The glass plates were placed over a $1.2 \mathrm{ml}$ deep well plate and $200 \mu$ l of elution buffer (10 mM Tris $\mathrm{pH} 8.0$, $20 \mu \mathrm{g} / \mathrm{ml}$ RNase) was added to each well. Finally, $200 \mu \mathrm{l}$ of eluted DNA was collected in $1.2 \mathrm{ml}$ deep well plate by centrifugation at 3,000 RPM for $5 \mathrm{~min}$. About 10-15 samples were randomly selected to check for concentration using the Qubit ${ }^{\circledR}$ HS DNA kit in the Qubit ${ }^{\circledR} 2.0$ fluorometer (Invitrogen, Carlsbad, CA, United States).

\section{PCR-GBS Library Preparation and Ion Torrent Sequencing}

A PCR-GBS SNP marker panel containing 365 barley SNP markers designed by Tamang (2017) was used in this study. The marker panels were divided into six pools as described by Tamang (2017). Six different PCR amplification reactions per sample, each representing one primer pool, were run for library preparation. Each reaction volume of $5 \mu \mathrm{l}$ contained $1.5 \mu \mathrm{l}$ of gDNA (DNA concentration ranging for $10-50 \mathrm{ng} / \mu \mathrm{l}), 1 \mu \mathrm{l}$ of $500 \mathrm{nM}$ primer pool and $2.5 \mu \mathrm{l}$ of $2 \times$ Platinum $^{\circledR}$ Multiplex PCR Master Mix (Life Technologies, CA, United States). The primary PCR amplifications were done in a Mastercycler Pro programmable thermocycler (Eppendorf, Hauppauge, NY, United States) with conditions set at: initial denaturation of $94^{\circ} \mathrm{C}$ for $10 \mathrm{~min}$; followed by a touchdown step of 10 cycles of denaturation at $94^{\circ} \mathrm{C}$ for $20 \mathrm{~s}$ and annealing at $62^{\circ} \mathrm{C}$ for $1 \mathrm{~min}$, where the temperature was decreased by $0.8^{\circ} \mathrm{C}$ each cycle; followed by 20 cycles of denaturation at $94^{\circ} \mathrm{C}$ for $20 \mathrm{~s}$, annealing at $57^{\circ} \mathrm{C}$ for $1 \mathrm{~min}$, and extension at $72^{\circ} \mathrm{C}$ for $1 \mathrm{~min}$; ending with a final template extension at $72^{\circ} \mathrm{C}$ for $3 \mathrm{~min}$. The PCR reactions were diluted to a volume of $20 \mu \mathrm{l}$ using nuclease-free $\mathrm{H}_{2} \mathrm{O}$. After brief vortexing and centrifugation, $5 \mu \mathrm{l}$ of diluted PCR product from each well was aliquoted to a new 96-well plate; each well represented a single sample with a total volume of $30 \mu$ per sample ( $5 \mu \mathrm{l} \times 6$ primer pools). A barcoding PCR mix of $20 \mu \mathrm{l}$ volume was prepared using $2 \mu \mathrm{l}$ of pooled single genotype amplicons, $1 \mu \mathrm{l}$ of $5 \mu \mathrm{M}$ unique barcode primers, $150 \mu \mathrm{M}$ of dNTPs, $250 \mathrm{nM}$ of universal reverse primer, $1 \times$ GoTaq $^{\circledR}$ Buffer (Promega, United States) and $1 \mathrm{U}$ of GoTaq ${ }^{\circledR}$ DNA polymerase. The PCR amplification parameters were the same as the primary PCR. After completion of secondary PCR cycles, $15 \mu$ l of nucleasefree water was added to each well followed by a quick vortex and spin. From each well, $5 \mu \mathrm{l}$ of samples were aliquoted and combined in a clean $1.5 \mathrm{ml}$ tube (total volume $=5 \mu \mathrm{l} \times$ total number of samples) and purified using a E.Z.N.A Cycle Pure Kit (Omega Bio-tek, Inc., GA, United States). An aliquot of $2 \mu \mathrm{l}$ from the purified pooled PCR amplicons was used for PCR-GBS library amplification in a $30 \mu \mathrm{l}$ reaction containing $1 \times \mathrm{GoTaq}^{\circledR}$ Buffer, $333 \mu \mathrm{M} \mathrm{ABC1}$ and P1 primer, $166 \mu \mathrm{M}$ dNTPs and $1 \mathrm{U}$ 
GoTaq $^{\circledR}$ DNA polymerase. A negative control reaction was set up without GoTaq ${ }^{\circledR}$ DNA polymerase. Both reactions were run in a PCR thermocycler with parameters set at: initial denaturation of $95^{\circ} \mathrm{C}$ for $5 \mathrm{~min}$; followed by eight cycles of denaturation at $95^{\circ} \mathrm{C}$ for $30 \mathrm{~s}$, annealing at $62^{\circ} \mathrm{C}$ for $30 \mathrm{~s}$, and extension at $72^{\circ} \mathrm{C}$ for $30 \mathrm{~s}$; ending with final template extension at $72^{\circ} \mathrm{C}$ for $7 \mathrm{~min}$. After PCR, the concentration of the Taq and NoTaq reactions were measured using the Qubit HS DNA kit with the Qubit 2.0 fluorometer. About $2 \mu \mathrm{l}$ of reaction were run on a $1 \%$ agarose gel pre-stained with Gelred (Biotium, Hayward, CA, United States) to visualize the amplicons. No spurious band $(<100 \mathrm{bp})$ were observed in the reaction with Taq and thus were processed for enrichment using Ion $\mathrm{PI}^{\mathrm{TM}} \mathrm{Hi}-\mathrm{Q}^{\mathrm{TM}}$ OT2 200 Kit (Life Technologies) without any further purification. The enrichment was done with the Ion OneTouch ${ }^{\text {TM }} 2$ System and finally, the samples were sequenced on the Ion Torrent Personal Genome Machine (PGM) using an Ion $318^{\mathrm{TM}}$ Chip following the manufacturers standard protocol.

\section{SNP Calling and Genotyping}

The sequencing reads generated with the Ion Torrent PGM were uploaded to CLC genomics workbench v8.0 software (CLC bio, Aarhus, Denmark) to perform the quality trimming (using default parameters) and end reads trimming. The 5' and 3' end of each sequence was trimmed by 22 nucleotides to remove the PCR adapters (Richards et al., 2016). The trimmed reads were used for alignment using the Burrows-Wheeler Aligner maximal exact match (BWA-MEM) algorithm (Li, 2013). The sequence for each marker was obtained from the barley T3 database ${ }^{1}$ and used to design a fasta reference file for mapping. The SNP calling was done on the aligned BAM (Binary Alignment Map) file using the Genome Analysis Toolkit (GATK) Unified Genotyper tool with default setting for multi-sample SNP calling (Van der Auwera et al., 2013). VCFtools was used to remove individual calls with read depth of less than 6 and genotype quality less than 10 (Danecek et al., 2011). Only the SNPs reported in the iSelect 9K SNP array for the selected markers were used for mapping.

An in-house visual basic script was utilized to calculate the frequency of the reference and alternate SNP allele called for each marker in each sample. The genotype of a sample for a given marker called as homozygous for reference allele or alternate allele, if the allele frequency of either of the alleles is greater than $70 \%$. Sample with allele frequency within $30-70 \%$ for either of the allele for a given SNP were scored as heterozygous.

\section{Genetic Mapping}

The iSelect consensus genetic map developed by MuñozAmatriaín et al. (2014) was used as a reference to assign the markers to their respective barley chromosome and chromosomal position. The genotypic and phenotypic data were used to manually construct a.qdf file containing a standard format for importing marker, map and trait data into the publicly available QTL mapping software QGene v.4.3.10 (Joehanes and Nelson, 2008). The imported data were then analyzed by a

${ }^{1}$ https://triticeaetoolbox.org/barley/ composite interval mapping (CIM, Jansen and Stam, 1994; Zeng, 1994) in QGene v.4.3.10. To control the background variation, a forward cofactor selection method was used to select marker as cofactors with options, "Maximum number of cofactor" and "F to add" set at auto. Permutations of 1,000 were used to obtain a LOD threshold for an experiment-wide significance level of 0.01 .

\section{Genetic Marker Saturation on Chromosome 5H Using PCR GBS}

To saturate the region delimiting Rrr 1 a total of 24 polymorphic (Q21861 and Pinnacle) iSelect markers located between the two chromosomes 5H markers 11_21247 and 12_30162 were selected. Genotypic data on Q21861 and Pinnacle were obtained from different genotypic experiments that are publicly available in the Barley T3 database (see footnote 1). For each marker, the forward and reverse primers were designed to amplify the region containing the diagnostic SNP (Supplementary Table S5). Adaptor sequences CS1 and CS2 were attached to the forward and reverse primers, respectively, for Ion Torrent sequencing compatibility (Richards et al., 2016). The primers were multiplexed into two pools, each containing 12 markers, as recommended by Richards et al. (2016). These new pools were used to re-genotype the PRN/Q21861 $\mathrm{F}_{4: 5}$ RILs and parental lines. The PCR-GBS library preparation, Ion Torrent sequencing, SNP calling and genetic mapping was performed as previously described. This genotypic data from the new set of markers on barley chromosome $5 \mathrm{H}$ was combined with the $5 \mathrm{H}$ marker data from the 365 marker panel.

The phenotypic score obtained from screening the $\mathrm{F}_{4: 5}$ RILs using Pgt race QCCJB and HKHJC was converted to a binary scale to match the genotypic calls [score $<=3=\mathrm{A}(\mathrm{Q} 21861$ like allele), score $>3=\mathrm{B}$ (Pinnacle like allele)] and used as the phenotypic markers Rrr1 and Rrr2, respectively. A genetic map was created using MapDisto v1.7.7.0.1.1 with default minimum LOD of 3.0, rmax of 0.3 and the Kosambi mapping function (Kosambi, 1943). In order to compute a physical distance between RMRL and the iSelect marker used in this mapping, their physical positions were obtained by conducting a blast search against IBSC (International Barley Sequencing Consortium) v1 Morex genome using Viroblast in the IBSC blast server (Deng et al., 2007; Mascher et al., 2017) ${ }^{2}$.

\section{Genetic Marker Saturation Using Barley 50K iSelect SNP Array}

The barley $50 \mathrm{~K}$ iSelect SNP array was used to genotype a total of 77 PRN/Q21861 $\mathrm{F}_{5: 6}$ RILs and their parental lines, Q21861 and Pinnacle-NIL to saturate the genetic map of barley chromosome $5 \mathrm{H}$ and $7 \mathrm{H}$. The genotyping was performed as described by Bayer et al. (2017). The genotypic data was filtered to obtained genetic markers with minor allele frequency (maf) $>0.1$ and missing data $<20 \%$. The filtered genotypic data from $50 \mathrm{~K}$ assay was combined with genotypic data from PCR-GBS to compute genetic map distance between markers in chromosome $5 \mathrm{H}$ and $7 \mathrm{H}$ using MapDisto v1.7.7.0.1.1 with default settings of

\footnotetext{
${ }^{2}$ http://webblast.ipk-gatersleben.de
} 
minimum LOD of 3.0, rmax of 0.3 and the Kosambi mapping function (Kosambi, 1943). The genetic map distances were utilized to select non-co-segregating markers. Finally, non-cosegregating markers belonging to barley chromosome $5 \mathrm{H}$ and $7 \mathrm{H}$, respectively, were used to compute genetic distances in MapDisto v1.7.7.0.1.1 using the aforementioned parameters. The genetic distances between selected markers were used to draw a linkage map for $5 \mathrm{H}$ and $7 \mathrm{H}$ using the publicly available software MapChart v2.32 (Voorrips, 2002).

\section{Candidate Genes Identification}

The publicly available high-confidence genes that were annotated for IBSC RefSeq v1.0 of barley cv. Morex genome ${ }^{3}$ were used to identify the candidate Rrr1 and Rrr2 genes within the delimited region. The physical position of the genetic markers delimiting the Rrr 1 and Rrr2 genes were used to define the physical contigs containing the candidate genes.

\section{RESULTS}

\section{Phenotypic Evaluation of NILs}

In the Njoro, Kenya Pgt race TTKSK inoculated field nursery, the RMRL donor parent Q21861 for the PRN, showed consistent resistance responses with a median IR of trace $(\mathrm{T})$ for disease severity and moderately resistant for IT (TMR; Supplementary Table S1). Disease rating data for the HQ1 NIL, the donor of the Q21861 RMRL for the CRN, was not produced at this site year, however, the previous site year screening of HQ1 in the same nursery showed a median IR of 5RMR. The IR for Pinnacle were clearly susceptible ranging from 15 to $30 \mathrm{MSS}$ with a median response of 25MSS. Likewise, Conlon gave a clear susceptible reaction with an IR ranging from $5 \mathrm{MS}$ to $25 \mathrm{MSS}$ and a median score of 20MS. Interestingly, a segregating disease response was observed for the $12 \mathrm{CRN}$ individuals tested. However, three of the CRN individuals (FAR14-94A-1, FAR14-94A-2, FAR14-95A2) were clearly resistant compared to the susceptible recurrent parent Conlon. FAR14-94A-1 gave a consistent disease response of 5MSMR across both replication, while FAR14-94A-2 had data from replication 2 only with a score of 5MR and FAR14-95A2 gave a consistent disease score of $5 \mathrm{MS}$ across all replications (Supplementary Table S1). However, all four individuals of PRN (FAR14-1-1, FAR14-1-2, FAR14-1-3 and FAR14-1-4) were susceptible with scores comparable to its susceptible parent Pinnacle (Supplementary Table S1). The highest disease score of 30MSS was observed in FAR14-1-2 and the lowest score of 10MSS was observed in FAR14-1-4.

Inoculation of resistance parental lines Q21861 and HQ1 with Pgt race QCCJB exhibited low ITs (Supplementary Table S2). The susceptible parental lines, Pinnacle and Conlon were both susceptible to QCCJB with a median score of 3-2 and 3,3-, respectively. CRN (FAR14-94A-1) inoculated with QCCJB exhibited a resistance response with a median IR of 1 ; while the PRN (FAR14-1-1) was as susceptible as Pinnacle wild type with a median score of 3,3- (Supplementary Table S2).

\footnotetext{
${ }^{3}$ http://webblast.ipk-gatersleben.de/barley_ibsc/downloads/
}

The RMRL donor of the PRN, Q21861 was also highly resistant to $\mathrm{HKHJC}$ as it contains the Rpg1 gene, exhibiting a median score of 0;1 (Supplementary Table S2). Likewise, the RMRL donor of the CRN, HQ1, exhibited a high median IT of 3-2 as it was selected to lack Rpg1. Pinnacle and Conlon were resistant to HKHJC as they are known to contain Rpg1. The CRN (FAR14-94A-1) as expected exhibited resistant ITs, median ITs of 1; similar to line Q21861. However, the PRN (FAR14-1-1) was different from both of its parents, exhibiting a highly susceptible median IT of 3-3.

Ninety-four of the PRN/Q21861 $\mathrm{F}_{2}$ individuals were inoculated with the RMRL specific Pgt race QCCJB. In this assay, consistent with previous assays of the parental lines, PRN and Q21861 exhibited median ITs of 3-2 and 0;1, respectively (Supplementary Table S3). Among the $\mathrm{F}_{2}$ progeny, the lowest ITs were 0; and the highest were 3+ (Supplementary Table S3). Likewise, the lowest observed IR in the $\mathrm{F}_{4: 5}$ RILs inoculated with QCCJB was 0; and the highest IR was 3, 3+ (Supplementary Table S4 and Figure 1A). The screening of $\mathrm{F}_{5: 6}$ RILs with race HKHJC resulted in the lowest IR of 0 ; and the highest IR of $3+$ (Supplementary Table S4 and Figure 1B).

The Pearson's chi-square $\left(\chi^{2}\right)$ goodness of fit test confirmed that the $F_{2}$ progeny derived from the PRN/Q21861 cross, which was fixed for the Q21861 RMRL, segregated for 3 resistant: 1 susceptible, when inoculated with Pgt race QCCJB, suggesting monogenic inheritance of a single dominant gene required for RMRL function (Table 1). This result was further validated using the $\mathrm{F}_{4: 5}$ RILs inoculated with QCCJB that did not significantly deviate from 1 resistant: 1 resistant to susceptible ratio (Figure 2) as expected for a single gene segregation in the RIL population [chi-square $\left(\chi^{2}\right) p$-value $>0.05$, Table 1]. The segregation ratio of IRs of the $\mathrm{F}_{5: 6}$ RIL population inoculated with $\mathrm{HKHJC}$ did not comply with 1:1 (resistance:susceptibility) but fit a 3:1 ratio (resistance:susceptibility) (Table 1). The 3:1 segregation ratio for resistance to susceptibility in the RIL population inoculated with Pgt race HKHJC suggested that two genes with a complementary gene function are required for functional Rpg1mediated resistance in the presence of a fixed RMRL.

\section{Genetic Mapping of Rrr1 and Rrr2}

As expected from the Pearson's chi-square $\left(\chi^{2}\right)$ goodness of fit test, a single gene required for rpg4-mediated resistance, designated Rrr1, was mapped to the telomeric region of barley chromosome $5 \mathrm{H}, \sim 5 \mathrm{cM}$ proximal to RMRL (Figure 3 and Table 2). The genotypic data obtained from the 365 PCRGBS SNP marker panel was utilized to map the Rrrl gene to a region of barley chromosome $5 \mathrm{H}$, flanked by the SNP markers 11_20551 (130.93 cM iSelect consensus map position) and 12_30162 (156.7 cM iSelect) based on the consensus map generated by Muñoz-Amatriaín et al. (2014). After saturating the delimited Rrr1 region using a custom PCR-GBS SNP panel designed from SNPs mined from the recently released barley $50 \mathrm{~K}$ iSelect markers, the Rrr1 region was further delimited to an $\sim 1.4$ cM region distal to iSelect marker 11_21018 (153.74 cM iSelect position) (Figures 3, 4). Even though all the markers used to construct the linkage map were from the chromosome $5 \mathrm{H}$, MapDisto generated two different chromosome $5 \mathrm{H}$ linkage 

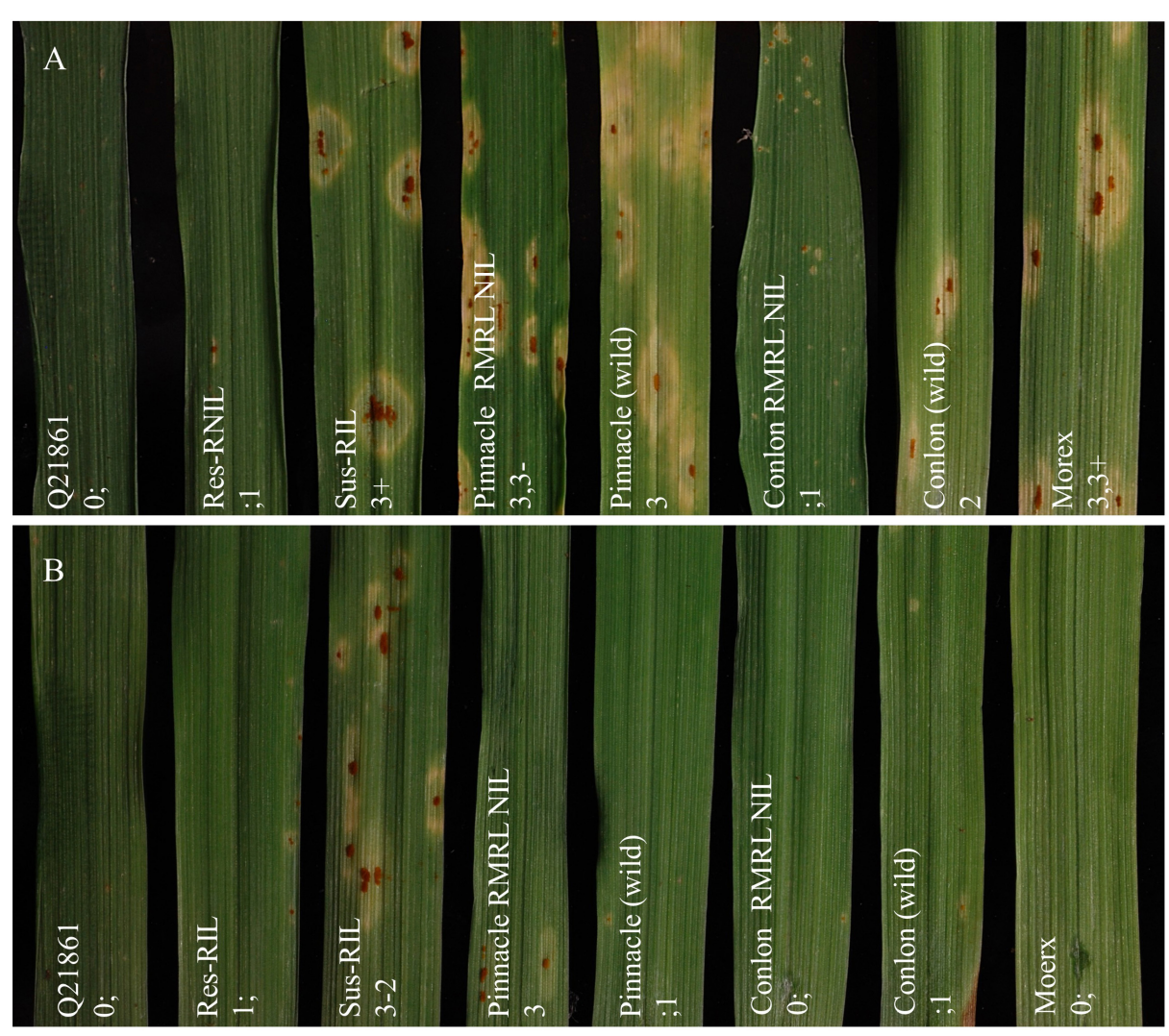

FIGURE 1 | Seedling assay using Puccinia graminis f. sp. tritici (Pgt) races QCCJB and HKHJC. (A) Pgt race QCCJB is avirulent on barley lines containing RMRL

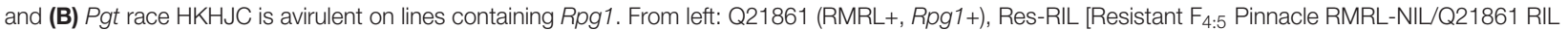

(RMRL+, Rpg1+)], Sus-RIL [Susceptible F 4:5 Pinnacle RMRL-NIL/Q21861 RIL (RMRL+, Rpg1+)], Pinnacle RMRL-NIL (RMRL+, Rpg1+), Pinnacle wild type (RMRL-, Rpg1+), Conlon RMRL-NIL (RMRL+, Rpg1+), Conlon wild type (RMRL-, Rpg1+), Morex (RMRL-, Rpg1+). A 0-4 modified scale for barley was used to score the disease response (Stakman et al., 1962; Steffenson et al., 2009).

groups due to the fixed Q21861 RMRL introgression in the PRN, which was monomorphic distal of Rrrl.

The genetic mapping of the genes segregating for Pgt race HKHJC resistance in the PRN/Q21861 RIL population as suspected by the 3:1 segregation ratio identified two genes/loci with duplicate dominant epistasis required for Rpg1-mediated resistance only in the presence of a fixed RMRL. One of the loci mapped co localized with Rrr1, while the other locus, the Rrr2 gene, mapped to the telomeric region of barley chromosome $7 \mathrm{H}$ (Figure 5), flanked by markers 11_21419 (0 cM iSelect position) and 12_30880 (63.28 cM iSelect position). This result confirmed that $R r r 1$ is required for RMRL function against $P g t$ races QCCJB and TTKSK mediated resistance. However, surprisingly Rrr 1 is also required for Rpg1-mediated resistance, but only in the presence of the RMRL, but Rrr2 can confer Rpg1 mediated resistance in the presence or abasence of Rrrl because of the duplicate gene action.

\section{Genetic Marker Saturation of Rrr1 and Rrr2 Using Barley iSelect 50K}

A total of 85 and 71 non-co-segregating markers were identified from the Barley iSelect $50 \mathrm{~K}$ data and utilized to saturate of barley chromosomes 5H Rrr1 and 7H Rrr2 loci, respectively. After marker saturation Rrr1 was delimited to $\sim 5.01 \mathrm{cM}$ based on recombination frequency of 77 PRN/Q21861 derived RILs between the flanking markers JHI-Hv50k-2016-350644 and JHIHv50k-2016-350801. The markers flanking Rrr1 delimited an $\sim 0.5 \mathrm{MB}$ physical region, slightly proximal $(\sim 1.8 \mathrm{MB})$ to RMRL on barley chromosome 5H (Table 2 and Figure 4A). Likewise, Rrr2 was delimited to an $\sim 1.27 \mathrm{cM}$ genetic region flanked by the markers JHI-Hv50k-2016-441751and JHI-Hv50k-2016442218 corresponding to a physical region of $\sim 0.7 \mathrm{MB}$, slightly distal ( 3.1 MB) to Rpg1 (Table 2 and Figure 5A). Based on the physical region delimiting Rrrl and Rrr2, 7 high-confidence genes were identified as candidate for Rrrl (Figure 4B and Supplementary Table S6); while 17 high-confidence genes were identified as Rrr2 candidates (Figure 5B and Supplementary Table S6).

\section{DISCUSSION}

The emergence of the highly virulent Pgt race TTKSK and subsequent wheat and barley yield losses in two continents due to epidemics caused by this race or its lineage leaves 
TABLE 1 | Chi-square goodness-of-fit test to assess the segregation for resistance to susceptibility in different generation of progenies generated from Pinnacle RMRL-NIL/Q21861.

\begin{tabular}{|c|c|c|c|c|c|c|c|}
\hline Pgt race & Generation $^{\mathrm{a}}$ & Experiment & Rep & \multicolumn{2}{|c|}{ No. of individuals ${ }^{b}$} & Expected segregation ratio ${ }^{c}$ & $p$-value ${ }^{d}$ \\
\hline \multirow[t]{4}{*}{ QCCJB } & $\mathrm{F}_{2}$ & 1 & 1 & 63 & 31 & $3: 1$ & 0.07 \\
\hline & $\mathrm{F}_{4: 5}$ & 1 & 1 & 81 & 38 & $1: 1$ & $8 \mathrm{E}-5$ \\
\hline & & & 2 & 75 & 44 & & 0.004 \\
\hline & & 2 & 1 & 50 & 61 & & 0.29 \\
\hline & & 2 & 1 & 79 & 30 & & 0.54 \\
\hline & & & 2 & 83 & 27 & & 0.91 \\
\hline
\end{tabular}

${ }^{a}$ The generation of progenies derived from Pinnacle RMRL-NIL/Q21861 cross that was screened with given Pgt race. ${ }^{b}$ The number of individuals that gave resistance or susceptible response when inoculated with given Pgt race. ${ }^{C}$ The expected Mendelian segregation ratio for resistance to susceptible in given assay. A trait governed by a single trait will segregate for 3:1 and 1:1 ratio in $F_{2}$ and RIL, respectively. In a RIL, two genes that are complementary for a single phenotype will give a phenotypic segregation 3:1 for resistance to susceptibility. ${ }^{d} p$-Value $>0.05$ represents that dataset that fit the expected Mendelian segregation ratio for resistance to susceptibility.

A
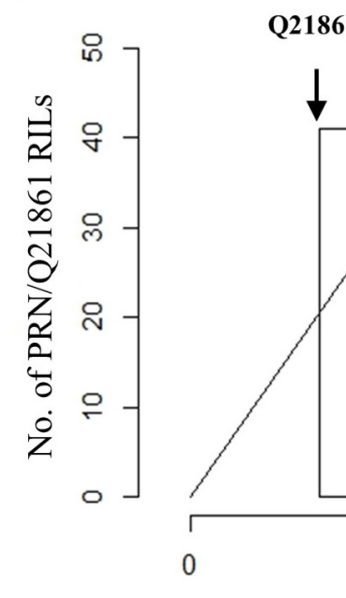

Q21861

Pinnacle RMRL-NIL

$\downarrow$

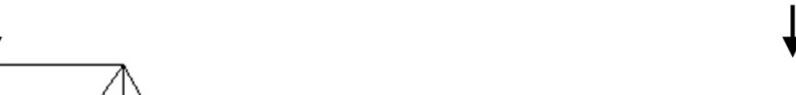

Q21861

Pinnacle RMRL-NIL

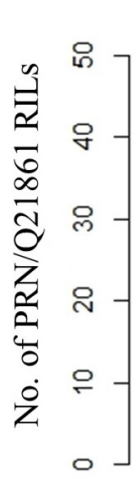

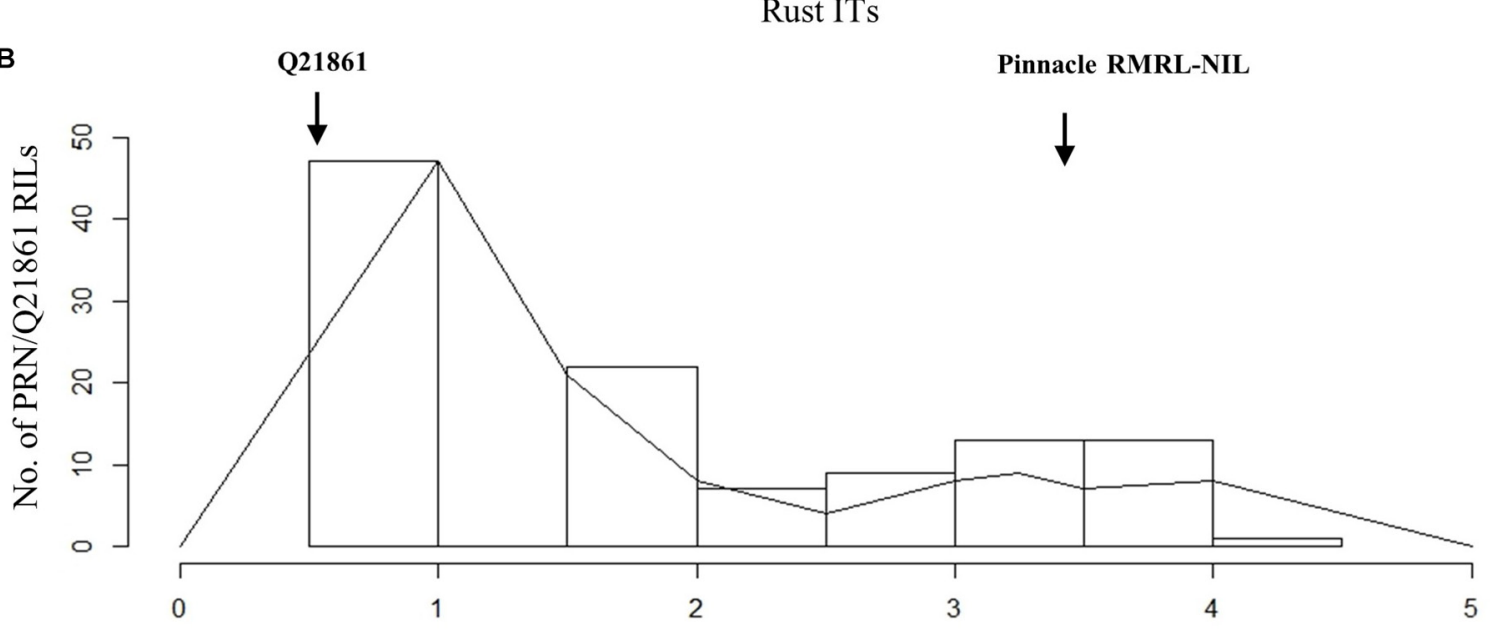

Rust ITs

FIGURE 2 | Histogram of stem rust infection types (ITs) exhibited by PRN/Q21861 RIL mapping population upon inoculation with Pgt races (A) QCCJB and (B) HKHJC. The rust ITs are shown on $y$-axis representing the quantitative scores obtained when using the conversion scale provided by Zhou et al. (2014). 
TABLE 2 | Genetic and physical location of markers associated with Rrr1 and Rrr2.

\begin{tabular}{|c|c|c|c|c|c|}
\hline iSelect marker & $\mathrm{Chr}^{\mathrm{a}}$ & $\mathrm{cM}^{\mathrm{b}}$ & $\mathrm{cM}^{\mathrm{c}}$ & Bp Start ${ }^{d}$ & Bp End ${ }^{d}$ \\
\hline JHI-Hv50k-2016-350644 & & N/A & N/A & 638453433 & 638453433 \\
\hline JHI-Hv50k-2016-350801 & & $\mathrm{N} / \mathrm{A}$ & N/A & 638951253 & 638951253 \\
\hline JHI-Hv50k-2016-351467 & & N/A & N/A & 640766892 & 640766892 \\
\hline $\mathrm{RMRL}^{\mathrm{e}}$ & & $\mathrm{N} / \mathrm{A}$ & N/A & 640765916 & 640837301 \\
\hline 11_21419 & $7 \mathrm{H}$ & 0 & N/A & 737146 & 737146 \\
\hline $\operatorname{Rpg} 1^{\dagger}$ & & N/A & N/A & 3257770 & 3451493 \\
\hline JHI-Hv50k-2016-441751 & & N/A & N/A & 5918682 & 5918682 \\
\hline JHI-Hv5Ok-2016-442218 & & $\mathrm{N} / \mathrm{A}$ & $\mathrm{N} / \mathrm{A}$ & 6623223 & 6623223 \\
\hline 12_30880 & & 63.28 & 54.39 & 67729137 & 67729137 \\
\hline
\end{tabular}

${ }^{a}$ Chromosome assignment based on iSelect consensus map (Muñoz-Amatriain et al., 2014) and POPSEQ map (Mascher et al., 2013) and the IBSC v2 barley genome sequence (Mascher et al., 2017). ${ }^{b}$ Chromosomal position based on iSelect consensus map developed by Muñoz-Amatriaín et al. (2014). ${ }^{\circ}$ Chromosomal position based on POPSEQ map developed by Mascher et al. (2013). 'Physical position in IBSC v1 genome sequence of barley cultivar Morex (Mascher et al., 2017). ${ }^{e}$ GenBank accession \# EU878778 used as a query for RMRL (Brueggeman et al., 2008). ' GenBank accession \# AF509750 used as a query for Rpg1 (Brueggeman et al., 2002).

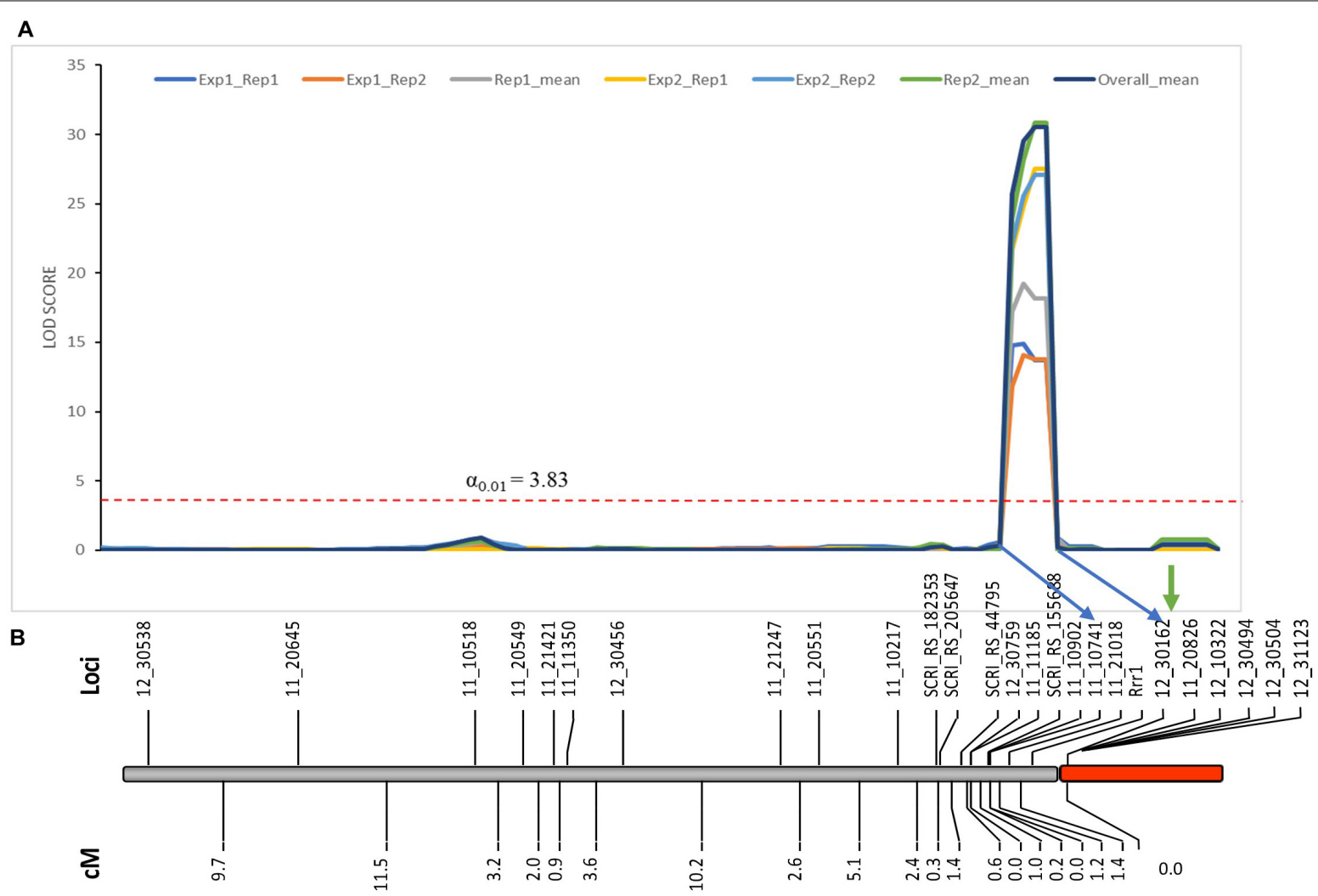

FIGURE 3 | (A) Genetic mapping of Rrr1 using the composite interval mapping in Qgene v4.3.0. on barley chromosome 5H. The dotted line represents a LOD threshold obtained at experiment-wide significance level of 0.01 after 1,000 permutations test. (B) The genetic map of barley chromosome $5 \mathrm{H}$ developed using the polymorphic markers available in our dataset. A default minimum LOD score of 3.0, rmax of 0.3 and Kosambi mapping function was used in MapDisto v1.7.7.0.1.1 to compute genetic map distance (cM) based on recombination frequency of 126 Q21861/Pinnacle-RMRL-NIL derived RILs. The PCR-GBS 365 marker panel contained no SNP markers distal to Rrr1 that were polymorphic between Q21861 and Pinnacle RMRL-NIL (represented by red color). The green arrow represents the position of RMRL [obtained using physical position (Table 2)].

production vulnerable if or when it is introduced to other regions (Singh et al., 2011; Steffenson et al., 2017). The only effective Pgt race TTKSK resistance in barley is the RMRL.
The RMRL contains three tightly linked genes, Rpg5, HvRga1 and $\operatorname{HvAdf3}$, which are inherited as a single genetic unit and function together for resistance (Brueggeman et al., 2008; 
A

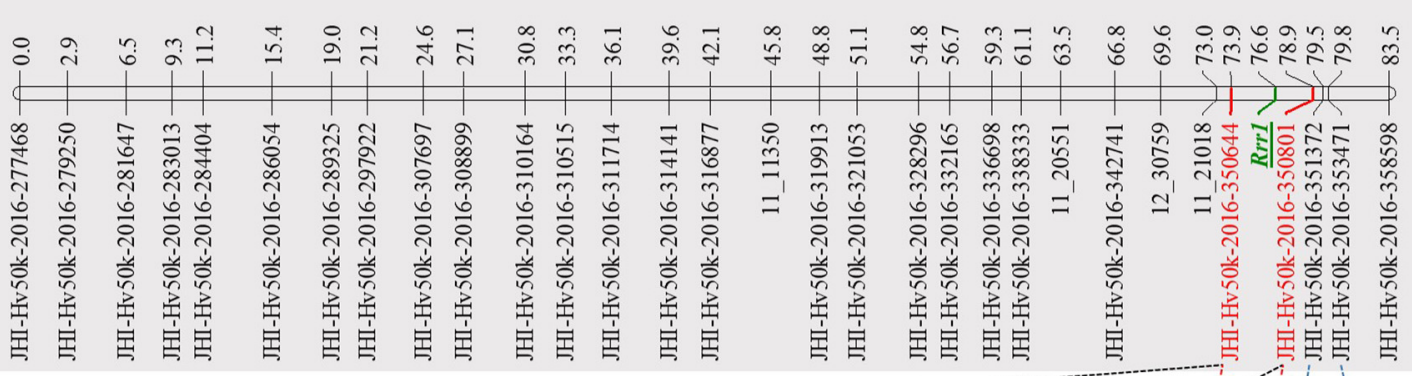

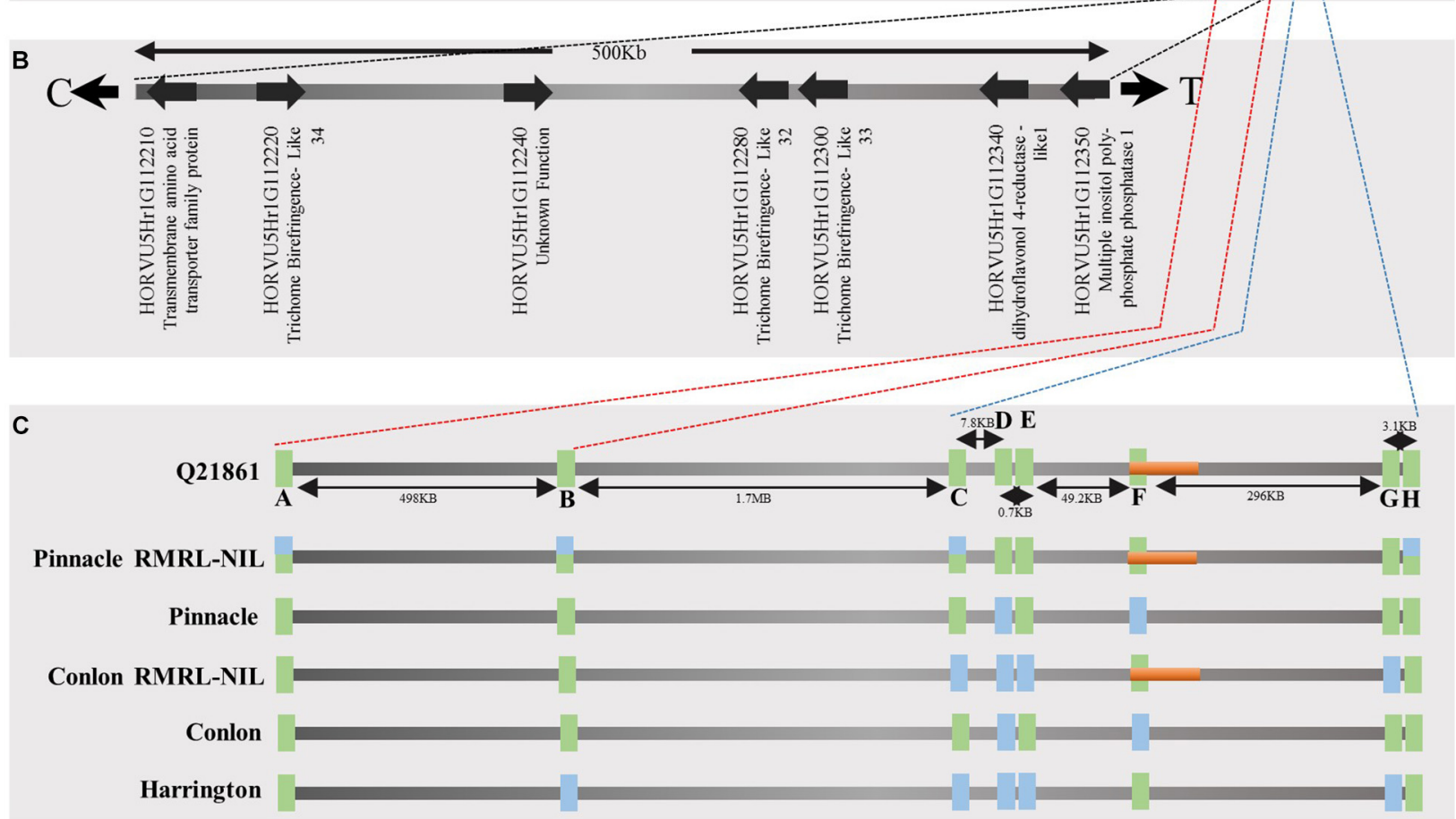

FIGURE 4 | Genetic marker saturation of barley chromosome 5H using barley 50K iSelect SNP array markers and identification of candidate genes for Rrr1. (A) The recombination frequency of 77 Q21861/Pinnacle RMRL-NIL derived RILs was used to compute genetic map distance between 84 non-co-segregating genetic markers and Rrr1 phenotypic markers using a Kosambi mapping function in MapDisto v.1.7.7.7. The genetic map distance was used to draw a linkage map in software MapChart v2.32. Rrr1 was delimited to a genetic distance of $~ 5.01$ cM between flanking markers JHI-Hv50K-2016-350644 and JHI-Hv50K-2016-350801. (B) The physical position of the genetic markers flanking Rrr1 was used to identify seven high-confidence annotate genes from IBSC RefSeq v1.0 of barley cv. Morex genome (http://webblast.ipk-gatersleben.de/barley_ibsc/downloads/). C and T at the end of the horizontal line indicate the direction of centromere and telomere, respectively. (C) Allele analysis of marker flanking Rrr1 (represented by red dotted lines) and RMRL (represented by blue dotted lines). 50K iSelect SNP markers are represented as A (JHI-Hv-50K-2016-350644), B (JHI-Hv-50K-2016-350801), C (JHI-Hv-50K-2016-351372), D (JHI-Hv-50K-2016-351438), E

(JHI-Hv-50K-2016-351449), F (JHI-Hv-5OK-2016-351467), G (JHI-Hv-50K-2016-351585) and H (JHI-Hv-50K-2016-351627). The green bars represent a SNP marker with a Q21861 allele and blue color represents the alternate allele, while semi-blue-green color represents a heterozygous call for a given marker. The orange bar represents a physical region containing RMRL.

Steffenson et al., 2009). Rpg5-specific markers have been used to efficiently introgress RMRL into elite barley (Derevnina et al., 2014) in several breeding programs, yet, it has been found to be ineffective when interacting with genes in some genetic backgrounds. We used Rpg5 allele specific molecular markers to incorporate the Q21861 RMRL into Upper Midwestern US malting varieties, developing NILs of the varieties Conlon and Pinnacle. Saturation of the NILs with SNP markers (Table 2 and Figure 4) defined the Q21861 introgression in the PRN and CRN validating successful RMRL introgression. However, only the CRN exhibited resistance to the RMRL specific Pgt races QCCJB and TTKSK comparable to Q21861. The PRN remained susceptible, suggesting the requirement of additional gene/s for resistance that are functionally polymorphic between the resistant barley accession Q21861 and the susceptible barley variety Pinnacle.

To identify the additional gene required for RMRL resistance, designated Rrr1 (required for $r p g 4$-mediated resistance 1), present in barley line Q21861, the PRN was crossed with Q21861 to develop a RIL mapping population that was fixed for RMRL and the Rpg1 stem rust resistance gene. Since, Q21861 and the PRN share identical Rpg1 and Rpg5 alleles, the loci segregating 


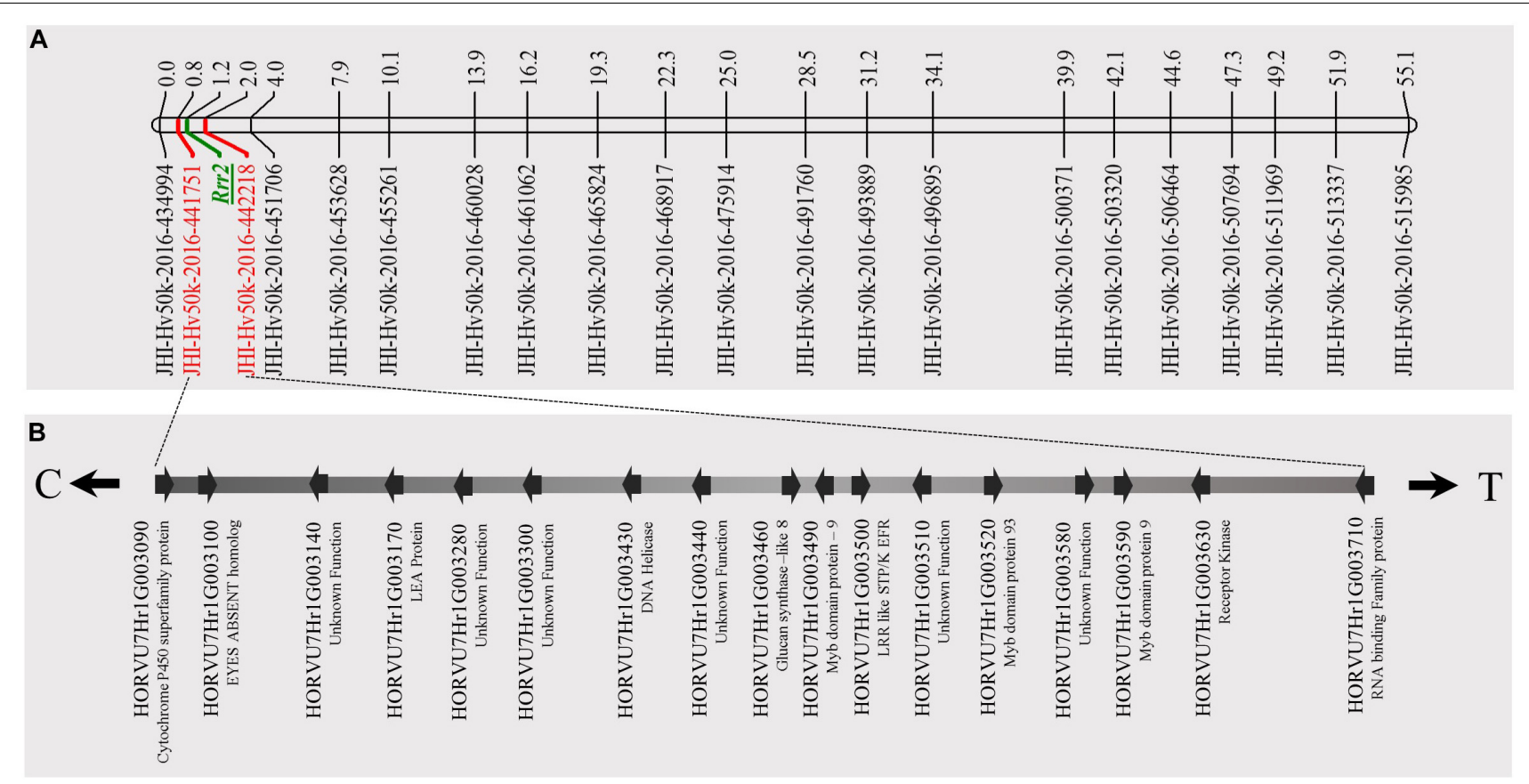

FIGURE 5 | Genetic map of barley chromosome 7H using the barley 50K iSelect SNP array markers and identification of candidate genes for Rrr2. (A) The genetic map distance of 71 non-co-segregating markers in barley chromosome $7 \mathrm{H}$ was computed based on the recombination frequency of 77 Q21861/Pinnacle RMRL-NIL derived using a Kosambi mapping function in MapDisto v.1.7.7.7. The publicly available software MapChart v2.32 was used to draw a linkage map using the obtained genetic map distances. Rrr2 was delimited to a genetic distance of $\sim 1.8 \mathrm{cM}$ between the flanking markers JHI-Hv50K-2016-441751 and JHI-Hv50K-2016-442218. (B) The physical position of the genetic markers flanking Rrr2 were used to identify seventeen high-confidence annotate genes from the IBSC RefSeq v1.0 of barley cv. Morex genome (http://webblast.ipk-gatersleben.de/barley_ibsc/downloads/). C and T at the end of the horizontal line indicate the direction of centromere and telomere, respectively.

for resistance/susceptibility in the RIL mapping population would be associated with any additional gene/s required for RMRL stem rust resistance. The responses to $P g t$ race QCCJB on the PRN/Q21861 RIL population segregated as a single dominant gene and mapped to the sub telomeric region of the long arm of barley chromosome $5 \mathrm{H}, \sim 5 \mathrm{cM}$ proximal to RMRL (Figures 3, 4). Because Rpg1 and rpg4 are distinct genes that independently assort on separate chromosomes, it was unexpected when we observed that the RMRL introgression into the Pinnacle background resulted in the suppression of Rpg1-mediated resistance against Pgt race HKHJC. However, because both Rpg1 and RMRL function together in the Q21861 background, we hypothesized that additional loci/genes from Q21861 should also segregate that allow for both resistances to function harmoniously in the same genetic background. The responses of Pgt race HKHJB on the RIL population segregated as two dominant genes ( 3 resistant: 1 susceptible) and one mapped to the Rrr1 region and the other mapped to the telomeric region of the short arm of barley chromosome 7H distal of Rpg1 and was designated $R r r 2$. These data determined that $R r r 1$ is required for RMRL function but is also required for Rpg1-mediated resistance but only in the presence of RMRL. However, the segregation ratio for resistance to susceptibility in response to HKHJB suggested that Rrr1 and Rrr2 complement each other for Rpg1-mediated resistance when RMRL is present (Figure 6 and Table 1). Thus, the PRN recovered both the non-functional allele of Rrr1 and
Rrr2 and failed to confer RMRL- or Rpg1-mediated stem rust resistance. Thus, it is apparent that Pinnacle and Q21861 have functionally polymorphic Rrr1 and Rrr2 alleles (Figure 4).

The data shows that the PRN contains non-functional rrrl and rrr2 alleles, thus Q21861 carries the dominant functional Rrr1 and Rrr2 alleles. Interestingly, Pinnacle confers Rpg1mediated resistance despite lacking the functional Rrr1 or Rrr2 suggesting that Rpg1-mediated resistance is independent of Rrr1 and Rrr2, in the absence of RMRL. The introgression of RMRL in barley genomes requires Rrrl to function and if RMRL is to be pyramided with Rpg1 then Rrrl must be present for Rpg1 to maintain its resistance. Although, when pyramiding these resistances to achieve the broad resistance like that found in line Q21861, which is effective against all known Pgt races, breeders must maintain Rrr1 in their lines. Interestingly, the Rrr2 gene can confer Rpg1-mediated resistance in the presence of RMRL, despite presence or absence of Rrrl, but does not complement RMRL function in the absence of Rrrl suggesting that these two genes do not have a complete duplicate dominant epistatic function. However, the complex suppression of Rpg1-mediated resistance by the presence of RMRL suggests that the two resistance mechanisms certainly have some level of interaction. This has also been exemplified by fast neutron irradiation-induced mutants that we developed with disrupted RMRL and Rpg1-mediated resistances (Unpublished). 


\begin{tabular}{|c|c|c|c|}
\hline & Haplotype & $\overline{\text { QCCJB }}$ & HKHJC \\
\hline Q21861 & $\begin{array}{l}\text { RMRL+, Rrr1+ } \\
\operatorname{Rpg} 1+, \operatorname{Rrr} 2+\end{array}$ & Resistant & Resistant \\
\hline Pinnacle & $\begin{array}{l}\text { rmrl-, rrr1- } \\
\operatorname{Rpg} 1+, \text { rrr2+ }\end{array}$ & Susceptible & Resistant \\
\hline Pinnacle NIL & $\begin{array}{l}\text { RMRL+, rrr1- } \\
\text { Rpg1+, rrr2- }\end{array}$ & Susceptible & Susceptible \\
\hline RIL-Type1 & $\begin{array}{l}\text { RMRL+, Rrr1+ } \\
\operatorname{Rpg} 1+, \operatorname{Rrr} 2+\end{array}$ & Resistant & Resistant \\
\hline RIL-Type2 & $\begin{array}{l}\text { RMRL+, rrr1- } \\
\operatorname{Rpg} 1+, \text { Rrr2+ }\end{array}$ & Susceptible & Resistant \\
\hline RIL-Type3 & $\begin{array}{l}\text { RMRL+, Rrr1+ } \\
\operatorname{Rpg} 1+, \text { rrr2- }\end{array}$ & Resistant & Resistant \\
\hline RIL-Type4 & $\begin{array}{l}\text { RMRL+, rrr1- } \\
\operatorname{Rpg} 1+, \operatorname{rrr} 2-\end{array}$ & Susceptible & Susceptible \\
\hline
\end{tabular}

FIGURE 6 | Seedling assay using Pgt race QCCJB and HKHJC showing disease response on barley containing different combination of RMRL, Rpg1, Rrr1 and Rrr2 alleles. QCCJB is virulent on Rpg1 and HKHJC is virulent on RMRL. Pinnacle is the recurrent parent and Q21861 is the RMRL donor of the Pinnacle RMRL-NIL. Four different allelic combinations of the two additional genes required for stem rust resistance, Rrr1 and Rrr2, occur in the Pinnacle RMRL-NIL/Q21861 progeny. Rrr1 is required to confer RMRL mediated QCCJB resistance (Q21861, RIL-Type1 and RIL-Type2). Rpg1 mediated resistance is independent of Rrr1 and Rrr2 in absence of RMRL (Pinnacle). The introgression of RMRL into the Pinnacle background necessitates the presence of either a functional Rrr1 or Rrr2 to confer Rpg1 mediated HKHJC resistance (Q21861, RIL-Type1, RIL-Type2, RIL-Type3). Rpg1 mediated HKHJC resistance in RIL-Type2 and RIL-Type3 shows that Rrr1 and Rrr2 have a complementary gene function in the Rpg1-mediated resistance pathway.

Genetic and mutational analysis of diverse pathosystems including barley, tomato and Arabidopsis have characterized genes that are quintessential for the function of $R$-gene mediated defense responses (Torp and Jørgensen, 1986; Freialdenhoven et al., 1994; Aarts et al., 1998; Feys and Parker, 2000; Glazebrook, 2001). Utilizing fast neutron irradiated Arabidopsis ecotype Col-1 mutations were produced in the non-race-specific disease resistance, NDR1, and enhanced disease susceptibility, EDS1 genes which showed their imperative roles in conferring disease resistance to biotrophic oomycetes and bacterial pathogens conferred by distinct classes of $R$ genes (Aarts et al., 1998; Li et al., 2001). Another study using barley mutants showed a role of two genes Rar1 and Rar2 in executing a race specific resistance by the gene Mla12 against the fungal pathogen Erysiphe graminis f. sp. hordei (Torp and Jørgensen, 1986; Freialdenhoven et al., 1994). A mutagenesis study in tomato identified a gene Rcr3 that is required for $C f-2$ mediated leaf mold resistance caused by Cladosporium fulvum (Dixon et al., 2000). However, many of these genes are highly conserved across genotypes unlike the Rrr 1 and Rrr2 genes, which certainly have functionally polymorphic alleles within the primary barley germplasm pool. Thus, many of these conserved genes could only be discovered by mutational studies that developed synthetic polymorphism that was not present in the primary germplasm pools.

A previous study utilized fast neutron irradiation of barley variety Morex generated the required for P. graminis resistance, Rpr1 mutant that disrupted Rpg1-mediated resistance (Zhang et al., 2006). Rpr1 was characterized utilizing a Q21861/Rpr1 mapping population which mapped the gene to a region of barley chromosome $4 \mathrm{H}$. Microarray analysis of the mutant and wild type identified three deleted genes in that region, and a putative serine/threonine protein kinase-like protein was considered as a strong candidate for Rpr1 gene. Another study by Gill et al. (2016) used gamma-irradiation to develop six independent 
mutants in the cv Morex background designated Rpr2-7 that also compromised Rpg1-mediated resistance. Utilizing a Q21861 $\mathrm{X} r p r 2 \quad \mathrm{~F}_{2}$ population Rpr2 was mapped to the centromeric region of chromosome $6 \mathrm{H}$. However, these studies did not report genes on chromosome $5 \mathrm{H}$ and $7 \mathrm{H}$ that function in stem rust resistance, thus are distinct from Rpr1 and Rpr2. Also, these studies utilized irradiation to produce artificial functional polymorphism suggesting that these genes are probably conserved resistance components. However, Rrr1 and $R r r 2$ are functionally polymorphic in the primary barley germplasm pool and more importantly in breeding programs; thus, our study is important to address issues that would arise when pyramiding Rpg1 and RMRL. It will be important to identify the Rrrl gene and identify diagnostic markers that could be used in combination with Rpg5 specific markers for effective marker assisted selection.

Polymerase chain reaction-GBS allowed us to utilize a larger number of RIL individuals to obtain a rough genetic map of the region containing Rrr1 and $R r r 2$. Then, the prior knowledge of RIL genotypes based on the PCR-GBS data allowed for the efficient use of the barley $50 \mathrm{~K}$ iSelect assay by selecting informative RIL individual to develop a genetic map with higher marker saturation. Utilizing the annotated barley genome sequence the physical region containing Rrrl was delimited to $\sim 0.5 \mathrm{Mb}$ containing seven $\mathrm{HC}$ genes and $R r r 2$ to $\sim 0.7 \mathrm{Mb}$ containing 17 HC genes (Supplementary Table S5). To prioritize the candidates Rrr1 and Rrr2 genes we searched for homologous or similar function genes since our mapping results suggests a somewhat duplicate dominant epistatic action of Rrr1 and Rrr2 in regard to Rpg1-mediated resistance in the presence of RMRL. Interestingly, this study identified gene families in Rrr1 and Rrr2 that are not homologous but have been shown to be involved in plant immune response through cell wall modifications. Out of the seven Rrrl candidate genes, three belong to the Trichrome birefringence like (TBL) family that function both as positive or negative regulators of plant defense responses depending upon the pathosystem. A powdery mildew resistance gene PMR5 (Powdery mildew resistant 5) was identified as a member of the TBL family that contributed to powdery mildew susceptibility, possibly through pectin acetylation (Vogel et al., 2004; Lim, 2013). In contrast, two TBL proteins in rice, OsTBL1 and OsTBL2, contributed to resistance against leaf blight through xylan modification (Gao et al., 2017). Likewise, a Glucan synthase-like 8 (GSL-8) Rrr2 candidate gene belongs to a GSL family that was shown to be involved in both positive and negative regulation of disease resistance in plants through cell wall modification (Nishimura et al., 2003; Ellinger and Voigt, 2014; Chowdhury et al., 2016). In Arabidopsis the powdery mildew resistance gene 4 (pmr4) is a mutant of the GSL-5 (AtGsl5) gene that was shown to enhanced resistance to the powdery mildew pathogen Golovinomyces cichoracearum (Nishimura et al., 2003). PMR4 was hypothesized to induce susceptibility by providing negative feedback for SA accumulation after callose induction upon early detection of the pathogen. Contrary to pmr4, the HvGsl6 (barley glucan synthase-like 6), a close homolog of $A t G s l 6$ was shown to reinforce resistance against barley powdery mildew Blumeria graminis $\mathrm{f}$. sp. hordei through callose deposition at the site of infection (Chowdhury et al., 2016). Since the genes belonging to TBL and GSL family have been shown to be involved in regulation of the plant immune response through cell wall modification and Rrr1 and Rrr2 have duplicate dominant gene action in Rpg1mediated stem rust resistance, it is possible that TBL is able to compensate the loss of GSL to restore Rpg1 resistance, despite TBL and GSL having different modes of cell wall modification. Based on this hypothesis, the genes belonging to TBL and GSL could be prioritized as top candidates for Rrr1 and Rrr2, respectively. However, we could not rule out the possibility of other genes as candidates for Rrr1 and Rrr2 especially other genes in the region with known defense function including the receptor like kinases present at the Rrr2 locus. Thus, post-transcriptional gene silencing and characterization of other stem rust resistance mutants are being utilized to validate the $R r r 1$ and $R r r 2$ candidate genes.

At the functional level plant immunity responses are typically characterized into either one of two tiers of the immunity responses. The first line of defense is known as pathogen-associated molecular pattern (PAMP)-triggered immunity (PTI) and the second and higher amplitude defense is known as effector-triggered immunity (ETI) (Jones and Dangl, 2006; Thomma et al., 2011). The PTI responses are activated early at the cell surface by membrane localized pattern recognition receptors (PRRs), which recognize conserved microbial molecules like bacterial flagellin or fungal chitin. ETI responses are typically later and generally rely on nucleotide binding-leucine-rich repeat (NLR) immunity receptors that recognize pathogen proteinaceous effectors that trigger resistance signaling pathways resulting in resistance responses. The ETI responses typically induce localized programmed cell death known as the hypersensitive response (HR). However, some resistance mechanisms are difficult to characterize under these definitions and two such mechanisms are the RMRL and Rpg1mediated resistance responses.

The stem rust resistance gene Rpgl was cloned and characterized by a positional cloning approach and shown to have an atypical R-protein structure consisting of tandem protein kinase domains (Brueggeman et al., 2002). Since the identification of Rpgl, it has been shown that $5 \mathrm{~min}$ post inoculation with avirulent Pgt race MCCF, the RPG1 protein is phosphorylated and degrades within $24 \mathrm{~h}$ and both modifications are required for resistance (Nirmala et al., 2010). The AVR-RPG1 effector proteins from the pathogen have since been identified and are present in the spore coat (Nirmala et al., 2011), suggesting that the interaction occurs prehaustoria formation. Interestingly, the RPG5 protein contains an STPK domain with homology to the RPG1 STPK gene family (Brueggeman et al., 2006) and may also elicit early defense responses similar to RPG1. However, the Rpg5 PK domain follows the "integrated decoy" or "integrated sensory domain" model (Cesari et al., 2014; Wu et al., 2015), which implies that it is possibly acted upon by an effector that is secreted by the pathogen and is possibly activated by an early recognition event as well. We hypothesize that both wheat stem rust resistance mechanisms in barley, Rpg1- and RMRL, rely on early prehaustorial recognition that possibly have some 
overlapping signaling mechanisms that when present together can inhibit each other in the absence of Rrr1 and/or Rrr2.

\section{AUTHOR CONTRIBUTIONS}

RS and RB designed the experiments, performed the stem rust assay, and wrote the manuscript. $\mathrm{RB}$ and $\mathrm{PG}$ developed the NIL and RIL populations. RS, TG, and AA-H constructed libraries for PCR-GBS and assisted in the experiments. RS did the bioinformatic and statistical data analysis, and genetic mapping.

\section{FUNDING}

This project was funded by National Science Foundation CAREER Grant No. 1253987. "Any opinions, findings, and

\section{REFERENCES}

Aarts, N., Metz, M., Holub, E., Staskawicz, B. J., Daniels, M. J., and Parker, J. E. (1998). Different requirements for EDS1 and NDR1 by disease resistance genes define at least two R gene-mediated signaling pathways in Arabidopsis. Proc. Natl. Acad. Sci. U.S.A. 95, 10306-10311. doi: 10.1073/pnas.95.17. 10306

Arora, D., Gross, T., and Brueggeman, R. (2013). Allele characterization of genes required for rpg4-mediated wheat stem rust resistance identifies Rpg5 as the R gene. Pythopathology 103, 1153-1161. doi: 10.1094/PHYTO-01-130030-R

Bayer, M. M., Rapazote-Flores, P., Ganal, M., Hedley, P. E., Macaulay, M., Plieske, A., et al. (2017). Development and evaluation of a Barley 50k iSelect SNP Array. Front. Plant Sci. 8:1792. doi: 10.3389/fpls.2017.01792

Borovkova, I. G., Steffenson, B. J., Jin, Y., Rasmussen, J. B., Kilian, A., Kleinhofs, A., et al. (1995). Identification of molecular markers linked to the stem rust resistance gene rpg4 in barley. Pythopathology 85, 181-185. doi: 10.1094/Phyto85-181

Brim, C. A. (1966). A modified pedigree method of selection in soybeans1. Crop Sci. 6, 220-220. doi: 10.2135/cropsci1966.0011183x000600020041x

Brueggeman, R., Drader, T., and Kleinhofs, A. (2006). The barley serine/threonine kinase gene Rpg1 providing resistance to stem rust belongs to a gene family with five other members encoding kinase domains. Theor. Appl. Genet. 113, 1147-1158. doi: 10.1007/s00122-006-0374-3

Brueggeman, R., Druka, A., Nirmala, J., Cavileer, T., Drader, T., Rostoks, N., et al. (2008). The stem rust resistance gene Rpg5 encodes a protein with nucleotidebinding-site, leucine-rich, and protein kinase domains. Proc. Natl. Acad. Sci. U.S.A. 105, 14970-14975. doi: 10.1073/pnas.0807270105

Brueggeman, R., Rostoks, N., Kudrna, D., Kilian, A., Han, F., Chen, J., et al. (2002). The barley stem rust-resistance gene Rpg1 is a novel disease-resistance gene with homology to receptor kinases. Proc. Natl. Acad. Sci. U.S.A. 99, 9328-9333. doi: 10.1073/pnas.142284999

Cesari, S., Bernoux, M., Moncuquet, P., Kroj, T., and Dodds, P. N. (2014). A novel conserved mechanism for plant NLR protein pairs: the "integrated decoy" hypothesis. Front. Plant Sci. 5:606. doi: 10.3389/fpls.2014.00606

Chowdhury, J., Schober, M. S., Shirley, N. J., Singh, R. R., Jacobs, A. K., Douchkov, D., et al. (2016). Down-regulation of the glucan synthase-like 6 gene (HvGsl6) in barley leads to decreased callose accumulation and increased cell wall penetration by Blumeria graminis f. sp. hordei. New Phytol. 212, 434-443. doi: $10.1111 /$ nph. 14086

Danecek, P., Auton, A., Abecasis, G., Albers, C. A., Banks, E., DePristo, M. A., et al. (2011). The variant call format and VCFtools. Bioinformatics 27, 2156-2158. doi: 10.1093/bioinformatics/btr330

Deng, W., Nickle, D. C., Learn, G. H., Maust, B., and Mullins, J. I. (2007). ViroBLAST: a stand-alone BLAST web server for flexible queries of multiple databases and user's datasets. Bioinformatics 23, 2334-2336. doi: 10.1093/ bioinformatics/btm 331 conclusions or recommendations expressed in this project are those of the author(s) and do not necessarily reflect the views of the National Science Foundation."

\section{ACKNOWLEDGMENTS}

We would like to thank Mary Osenga of USDA-ARS Small Grain Genotyping Centre in Fargo for performing iSelect Assay.

\section{SUPPLEMENTARY MATERIAL}

The Supplementary Material for this article can be found online at: https://www.frontiersin.org/articles/10.3389/fpls.2018.01789/ full\#supplementary-material

Derevnina, L., Fetch, T., Singh, D., Brueggeman, R., Dong, C., and Park, R. F. (2014). Analysis of stem rust resistance in Australian barley cultivars. Plant Dis. 98, 1485-1493. doi: 10.1094/PDIS-11-13-1174-RE

Dixon, M. S., Golstein, C., Thomas, C. M., Van der Biezen, E. A., and Jones, J. D. (2000). Genetic complexity of pathogen perception by plants: the example of Rcr3, a tomato gene required specifically by Cf-2. Proc. Natl. Acad. Sci. U.S.A. 97, 8807-8814. doi: 10.1073/pnas.97.16.8807

Ellinger, D., and Voigt, C. A. (2014). Callose biosynthesis in Arabidopsis with a focus on pathogen response: what we have learned within the last decade. Ann. Bot. 114, 1349-1358. doi: 10.1093/aob/mcu120

Feys, B. J., and Parker, J. E. (2000). Interplay of signaling pathways in plant disease resistance. Trends Genet. 16, 449-455. doi: 10.1016/S0168-9525(00)02 107-7

Freialdenhoven, A., Scherag, B., Hollricher, K., Collinge, D. B., ThordalChristensen, H., and Schulze-Lefert, P. (1994). Nar-1 and Nar-2, two loci required for Mla12-specified race-specific resistance to powdery mildew in barley. Plant Cell 6, 983-994. doi: 10.1105/tpc.6.7.983

Gao, Y., He, C., Zhang, D., Liu, X., Xu, Z., Tian, Y., et al. (2017). Two trichome birefringence-like proteins mediate xylan acetylation, which is essential for leaf blight resistance in rice. Plant Physio. 173, 470-481. doi: 10.1104/pp.16. 01618

Gill, U., Brueggeman, R., Nirmala, J., Chai, Y., Steffenson, B., and Kleinhofs, A. (2016). Molecular and genetic characterization of barley mutants and genetic mapping of mutant rpr2 required for Rpg1-mediated resistance against stem rust. Theor. Appl. Genet. 129, 1519-1529. doi: 10.1007/s00122-0162721-3

Glazebrook, J. (2001). Genes controlling expression of defense responses in Arabidopsis - 2001 status. Curr. Opin. Plant Biol. 4, 301-308. doi: 10.1016/ S1369-5266(00)00177-1

Harvey, B. L., and Rossnagel, B. G. (1984). Harrington barley. Can. J. Plant Sci. 64, 193-194. doi: 10.4141/cjps84-024

Horsley, R. D., Franckowiak, J. D., and Schwarz, P. B. (2009). "Barley," in Cereals, ed. M. J. Carena (New York, NY: Springer-Verlag), 227-250. doi: 10.1007/9780-387-72297-9_7

Ivanova, N. V., Fazekas, A. J., and Hebert, P. D. (2008). Semi-automated, membrane-based protocol for DNA isolation from plants. Plant Mol. Biol. Rep. 26, 186-198. doi: 10.1007/s11105-008-0029-4

Jansen, R. C., and Stam, P. (1994). High resolution of quantitative traits into multiple loci via interval mapping. Genetics 136, 1447-1455.

Jin, Y., Steffenson, B. J., and Fetch, T. G. (1994a). Sources of resistance to pathotype QCC of Puccinia graminis f. sp. tritici in barley. Crop Sci. 34, 285-288. doi: 10.2135/cropsci1994.0011183X00340001 0052x

Jin, Y., Steffenson, B. J., and Miller, J. D. (1994b). Inheritance of resistance to pathotypes QCC and MCC of Puccinia graminis f. sp. tritici in barley line Q21861 and temperature effects on the expression of resistance. Pathology 84, $452-455$. 
Jin, Y., Szabo, L. J., Pretorius, Z. A., Singh, R. P., Ward, R., and Fetch, T. Jr. (2008). Detection of virulence to resistance gene Sr24 within race TTKS of Puccinia graminis f. sp. Tritici. Plant Dis. 92, 923-926. doi: 10.1094/PDIS-92-6-0923

Joehanes, R., and Nelson, J. C. (2008). QGene 4.0, an extensible Java QTL-analysis platform. Bioinformatics 24, 2788-2789. doi: 10.1093/bioinformatics/btn523

Jones, J. D., and Dangl, J. L. (2006). The plant immune system. Nature 444, 323-329. doi: 10.1038/nature05286

Kleinhofs, A., Kilian, A., Maroof, M. S., Biyashev, R. M., Hayes, P., Chen, F. Q., et al. (1993). A molecular, isozyme and morphological map of the barley (Hordeum vulgare) genome. Theor. Appl. Genet. 86, 705-712. doi: 10.1007/BF0022 2660

Kosambi, D. D. (1943). The estimation of map distances from recombination values. Ann. Eugen. 12, 172-175. doi: 10.1111/j.1469-1809.1943.tb02 321.x

Li, H. (2013). Aligning sequence reads, clone sequences and assembly contigs with BWA-MEM. arXiv:1303.3997v1 [Preprint].

Li, X., Clarke, J. D., Zhang, Y., and Dong, X. (2001). Activation of an EDS1mediated R-gene pathway in the sncl mutant leads to constitutive, NPR1independent pathogen resistance. Mol. Plant Microbe Interact. 14, 1131-1139. doi: 10.1094/MPMI.2001.14.10.1131

Lim, C. C. (2013). Roles of the Plant Cell Wall in Powdery Mildew Disease Resistance in Arabidopsis thaliana: PMR5 (POWDERY MILDEW RESISTANT 5) Affects the Acetylation of Cell Wall Pectin, Doctoral dissertation, University of California, Berkeley, Berkeley, CA.

Mascher, M., Gundlach, H., Himmelbach, A., Beier, S., Twardziok, S. O., Wicker, T., et al. (2017). A chromosome conformation capture ordered sequence of the barley genome. Nature 544, 427-433. doi: 10.1038/nature22043

Mascher, M., Muehlbauer, G. J., Rokhsar, D. S., Chapman, J., Schmutz, J., Barry, K., et al. (2013). Anchoring and ordering NGS contig assemblies by population sequencing (POPSEQ). Plant J. 76, 718-727. doi: 10.1111/tpj. 12319

McIntosh, R. A., Wellings, C. R., and Park, R. F. (1995). Wheat Rusts: An Atlas of Resistance Genes. Clayton, VIC: Csiro Publishing. doi: 10.1007/978-94-0110083-0

Muñoz-Amatriaín, M., Cuesta-Marcos, A., Endelman, J. B., Comadran, J., Bonman, J. M., Bockelman, H. E., et al. (2014). The USDA barley core collection: genetic diversity, population structure, and potential for genome-wide association studies. PLoS One 9:e94688. doi: 10.1371/journal.pone.0094688

Nirmala, J., Drader, T., Chen, X., Steffenson, B., and Kleinhofs, A. (2010). Stem rust spores elicit rapid RPG1 phosphorylation. Mol. Plant Microbe Interact. 23, 1635-1642. doi: 10.1094/MPMI-06-10-0136

Nirmala, J., Drader, T., Lawrence, P. K., Yin, C., Hulbert, S., Steber, C. M., et al. (2011). Concerted action of two avirulent spore effectors activates Reaction to Puccinia graminis 1 (Rpg1)-mediated cereal stem rust resistance. Proc. Natl. Acad. Sci. U.S.A. 108, 14676-14681. doi: 10.1073/pnas.1111771108

Nishimura, M. T., Stein, M., Hou, B. H., Vogel, J. P., Edwards, H., and Somerville, S. C. (2003). Loss of a callose synthase results in salicylic acid-dependent disease resistance. Science 301, 969-972. doi: 10.1126/science.1086716

Peterson, R. F., Campbell, A. B., and Hannah, A. E. (1948). A diagrammatic scale for estimating rust intensity on leaves and stems of cereals. Can. J. Plant Sci. 26, 496-500. doi: 10.1139/CJPS-2018-0084

Pretorius, Z. A., Singh, R. P., Wagoire, W. W., and Payne, T. S. (2000). Detection of virulence to wheat stem rust resistance gene Sr31 in Puccinia graminis $\mathrm{f} . \mathrm{sp}$. tritici in Uganda. Plant Dis. 84:203. doi: 10.1094/PDIS.2000.84.2.203B

Richards, J., Chao, S., Friesen, T., and Brueggeman, R. (2016). Fine mapping of the barley chromosome $6 \mathrm{H}$ net form net blotch susceptibility locus. G3 6, 1809-1818. doi: 10.1534/g3.116.028902

Roelfs, A. P. (1992). Rust Diseases of Wheat: Concepts and Methods of Disease Management. Texcoco: Cimmyt.

Roelfs, A. P., Casper, D. H., Long, D. L., and Roberts, J. J. (1991). Races of Puccinia graminis in the United States in 1989. Plant Dis. 75, 1127-1130. doi: 10.1094/ PHYTO.1997.87.9.910

Roelfs, A. P., Long, D. L., and Roberts, J. J. (1993). Races of Puccinia graminis in the United States during 1990. Plant Dis. 77, 125-128. doi: 10.1094/PD-77-0125

Singh, R. P., Hodson, D. P., Huerta-Espino, J., Jin, Y., Bhavani, S., Njau, P., et al. (2011). The emergence of Ug99 races of the stem rust fungus is a threat to world wheat production. Annu. Rev. Pythopath. 49, 465-481. doi: 10.1146/annurevphyto-072910-095423
Singh, R. P., Hodson, D. P., Jin, Y., Huerta-Espino, J., Kinyua, M. G., Wanyera, R., et al. (2006). Current status, likely migration and strategies to mitigate the threat to wheat production from race Ug99 (TTKS) of stem rust pathogen. CAB Rev. 1, 1-13. doi: 10.1079/PAVSNNR20061054

Stakman, E. C., Stewart, D. M., and Loegering, W. Q. (1962). Identification of physiologic races of Puccinia graminis var. tritici. U.S. Agric. Res. Serv. 617, 1-53.

Steffenson, B. J. (1992). Analysis of durable resistance to stem rust in barley. Euphytica 63, 153-167. doi: 10.1038/nbt.3543

Steffenson, B. J., Case, A. J., Pretorius, Z. A., Coetzee, V., Kloppers, F. J., Zhou, H., et al. (2017). Vulnerability of barley to African pathotypes of Puccinia graminis f. sp. tritici and sources of resistance. Pythopathology 107, 950-962. doi: 10.1094/ PHYTO-11-16-0400-R

Steffenson, B. J., and Jin, Y. (2006). Resistance to race TTKS of Puccinia graminis $\mathrm{f}$. sp. tritici in barley. Pythopathology 96:S110.

Steffenson, B. J., Jin, Y., Brueggeman, R. S., Kleinhofs, A., and Sun, Y. (2009). Resistance to stem rust race TTKSK maps to the rpg4/Rpg5 complex of chromosome 5H of barley. Pythopathology 99, 1135-1141. doi: 10.1094/ PHYTO-99-10-1135

Sun, Y., Steffenson, B. J., and Jin, Y. (1996). Genetics of resistance to Puccinia graminis f. sp. secalis in barley line Q21861. Pythopathology 86, 1299-1302. doi: 10.1094/Phyto- 86-1299

Tamang, P. (2017). Genetic Mapping and Characterization of Net Blotch Dominant Resistance and Dominant Susceptibility Loci in Barley, Doctoral dissertation, North Dakota State University, Fargo, ND.

Thomma, B. P., Nürnberger, T., and Joosten, M. H. (2011). Of PAMPs and effectors: the blurred PTI-ETI dichotomy. Plant Cell 23, 4-15. doi: 10.1105/tpc.110. 082602

Torp, J., and Jørgensen, J. H. (1986). Modification of barley powdery mildew resistance gene Ml-a12 by induced mutation. Can. J. Genet. Cytol. 28, 725-731. doi: $10.1139 / \mathrm{g} 86-102$

United States Department of Agriculture [USDA] (2012). North Dakota 2012 Barley Varieties. Washington, DC: National Agricultural Statistics Service, USDA.

Van der Auwera, G. A., Carneiro, M. O., Hartl, C., Poplin, R., del Angel, G., LevyMoonshine, A., et al. (2013). From FastQ data to high-confidence variant calls: the genome analysis toolkit best practices pipeline. Curr. Prot. Bioinformatics 43, 11.10.1-11.10.33. doi: 10.1002/0471250953.bi1110s43

Vogel, J. P., Raab, T. K., Somerville, C. R., and Somerville, S. C. (2004). Mutations in PMR5 result in powdery mildew resistance and altered cell wall composition. Plant J. 40, 968-978. doi: 10.1111/j.1365-313X.2004.02 264.x

Voorrips, R. E. (2002). MapChart: software for the graphical presentation of linkage maps and QTLs. J. Hered. 93, 77-78. doi: 10.1094/MPMI-06-120146-R

Wang, X., Richards, J., Gross, T., Druka, A., Kleinhofs, A., Steffenson, B., et al. (2013). The rpg4-mediated resistance to wheat stem rust (Puccinia graminis) in barley (Hordeum vulgare) requires Rpg5, a second NBS-LRR gene, and an actin depolymerization factor. Mol. Plant Microbe Interact. 26, 407-418. doi: 10.1094/MPMI-06-12-0146-R

Wu, C. H., Krasileva, K. V., Banfield, M. J., Terauchi, R., and Kamoun, S. (2015). The "sensor domains" of plant NLR proteins: more than decoys? Front. Plant Sci. 6:134. doi: 10.3389/fpls.2015.00134

Yu, L. X., Lorenz, A., Rutkoski, J., Singh, R. P., Bhavani, S., Huerta-Espino, J., et al. (2011). Association mapping and gene-gene interaction for stem rust resistance in CIMMYT spring wheat germplasm. Theor. Appl. Genet. 123, 1257-1268. doi: 10.1007/s00122-011-1664-y

Zadoks, J. C., Chang, T. T., and Konzak, C. F. (1974). A decimal code for the growth stages of cereals. Weed Res. 14, 415-421.

Zeng, Z. B. (1994). Precision mapping of quantitative trait loci. Genetics 136, 1457-1468. doi: 10.1007/s00122-006-0342-y

Zhang, L., Fetch, T., Nirmala, J., Schmierer, D., Brueggeman, R., Steffenson, B., et al. (2006). Rprl, a gene required for Rpg1-dependent resistance to stem rust in barley. Theor. Appl. Genet. 113, 847-855. doi: 10.1007/s00122-0142297-8

Zhou, H., Steffenson, B. J., Muehlbauer, G., Wanyera, R., Njau, P., and Ndeda, S. (2014). Association mapping of stem rust race TTKSK resistance in US barley breeding germplasm. Theor. Appl. Genet. 127, 1293-1304. doi: 10.1007/s00122014-2297-8 
Zurn, J. D., Newcomb, M., Rouse, M. N., Jin, Y., Chao, S., Sthapit, J., et al. (2014). High-density mapping of a resistance gene to Ug99 from the Iranian landrace PI 626573. Mol. Breed. 34, 871-881.

Conflict of Interest Statement: The authors declare that the research was conducted in the absence of any commercial or financial relationships that could be construed as a potential conflict of interest.
Copyright $\odot 2018$ Sharma Poudel, Al-Hashel, Gross, Gross and Brueggeman. This is an open-access article distributed under the terms of the Creative Commons Attribution License (CC BY). The use, distribution or reproduction in other forums is permitted, provided the original author(s) and the copyright owner(s) are credited and that the original publication in this journal is cited, in accordance with accepted academic practice. No use, distribution or reproduction is permitted which does not comply with these terms. 